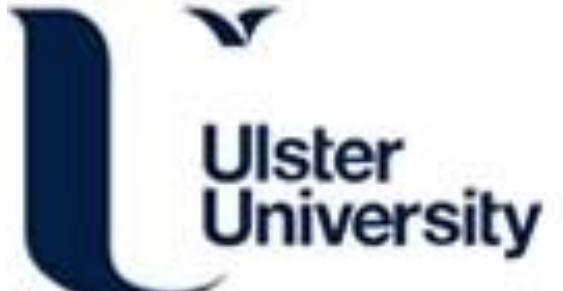

\section{Adsorption of Sophorolipid Biosurfactants on Their Own and Mixed with Sodium Dodecyl Benzene Sulfonate, at the Air/Water Interface}

Chen, ML., Dong, CC., Penfold, J., Thomas, RK., Smyth, TJP., Perfumo, A., Marchant, R., Banat, IM., Stevenson, P., Parry, A., Tucker, I., \& Campbell, RA. (2011). Adsorption of Sophorolipid Biosurfactants on Their Own and Mixed with Sodium Dodecyl Benzene Sulfonate, at the Air/Water Interface. Langmuir, 27, 8854-8866. https://doi.org/10.1021/la201660n

Link to publication record in Ulster University Research Portal

Published in:

Langmuir

Publication Status:

Published (in print/issue): 09/06/2011

DOI:

10.1021/la201660n

Document Version

Publisher's PDF, also known as Version of record

\section{General rights}

Copyright for the publications made accessible via Ulster University's Research Portal is retained by the author(s) and / or other copyright owners and it is a condition of accessing these publications that users recognise and abide by the legal requirements associated with these rights.

\section{Take down policy}

The Research Portal is Ulster University's institutional repository that provides access to Ulster's research outputs. Every effort has been made to ensure that content in the Research Portal does not infringe any person's rights, or applicable UK laws. If you discover content in the Research Portal that you believe breaches copyright or violates any law, please contact pure-support@ulster.ac.uk. 


\title{
Adsorption of Sophorolipid Biosurfactants on Their Own and Mixed with Sodium Dodecyl Benzene Sulfonate, at the Air/Water Interface
}

\author{
Minglei Chen, ${ }^{\dagger}$ Chuchuan Dong, ${ }^{\dagger}$ Jeff Penfold, $^{*,+, \neq}$ Robert K. Thomas, $^{\dagger}$ Thomas J. P. Smyth, $^{\S}$ \\ Amedea Perfumo, ${ }^{\S}$ Roger Marchant, ${ }^{\S}$ Ibrahim M. Banat, ${ }^{\S}$ Paul Stevenson, " Alyn Parry," Ian Tucker," and \\ Richard A. Campbell ${ }^{\perp}$ \\ ${ }^{\dagger}$ Physical and Theoretical Chemistry Laboratory, University of Oxford, South Parks Road, Oxford, United Kingdom \\ ${ }^{\ddagger}$ ISIS, STFC, Rutherford Appleton Laboratory, Chilton, Didcot, OXON, United Kingdom \\ ${ }^{\S}$ School of Biomedical Sciences, University of Ulster, Coleraine, Northern Ireland \\ "Unilever Research and Development Laboratory, Port Sunlight, Wirral, United Kingdom \\ ${ }^{\perp}$ Institut Laue Langevin, 6 rue Jules Horowitz, F-38042 Grenoble, Cedex 09, France
}

Supporting Information

ABSTRACT: The adsorption of the lactonic (LS) and acidic (AS) forms of sophorolipid and their mixtures with the anionic surfactant sodium dodecyl benzene sulfonate (LAS) has been measured at the air/water interface by neutron reflectivity, NR. The AS and LS sophorolipids adsorb with Langmuir-like adsorption isotherms. The more hydrophobic LS is more surface active than the AS, with a lower critical micellar concentration, $\mathrm{CMC}$, and stronger surface adsorption, with an area/molecule $\sim 70 \AA^{2}$ compared with $85 \AA^{2}$ for the AS. The
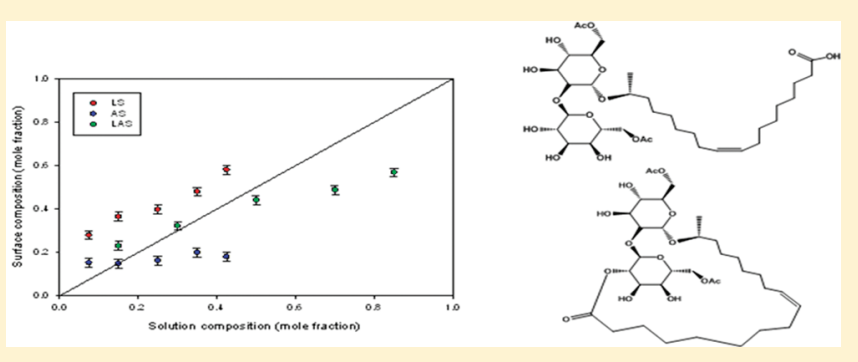
acidic sophorolipid shows a maximum in its adsorption at the CMC which appears to be associated with a mixture of different isomeric forms. The binary LS/AS and LS/LAS mixtures show a strong surface partitioning in favor of the more surface active and hydrophobic LS component but are nevertheless consistent with ideal mixing at the interface. In contrast, the surface composition of the AS/LAS mixture is much closer to the solution composition, but the surface mixing is nonideal and can be accounted for by regular solution theory, RST. In the AS/LS/LAS ternary mixtures, the surface adsorption is dominated by the sophorolipid, and especially the LS component, in a way that is not consistent with the observations for the binary mixtures. The extreme partitioning in favor of the sophorolipid for the LAS/LS/AS (1:2) mixtures is attributed to a reduction in the packing constraints at the surface due to the AS component. Measurements of the surface structure reveal a compact monolayer for LS and a narrow solvent region for LS, LS/AS, and LS/LAS mixtures, consistent with the more hydrophobic nature of the LS component. The results highlight the importance of the relative packing constraints on the adsorption of multicomponent mixtures, and the impact of the lactonic form of the sophorolipid on the adsorption of the sophorolipid/LAS mixtures.

\section{INTRODUCTION}

The use of synthetic petroleum based surfactants is under increasing pressure due to their unfavorable environmental impact. Biosurfactants are naturally produced by microorganisms as secondary metabolites, ${ }^{1,2}$ and their exact physiological roles are not yet fully understood. It is generally considered that they are produced to promote the growth of the microorganisms on waterimmiscible hydrophobic substrates by reducing the surface tension at the phase boundary thus making the hydrophobic substrate more readily available for uptake and metabolism. ${ }^{3-5}$ Biosurfactants are complex amphiphilic molecules whose polar and nonpolar domains depend on the carbon substrate available and the particular bacterial strain that has produced them. ${ }^{6}$ Their surface activity properties are broadly similar to conventional surfactants, resulting in reduced surface/interfacial tension, wetting, and emulsification. ${ }^{7}$ However, they have a number of key advantages over their synthetic counterparts, which include lower toxicity, antimicrobial properties, greater biodegradability, ${ }^{8}$ better environmental compatibility, ${ }^{9}$ high selectivity and specific activity at extreme temperatures, $\mathrm{pH}$, and salinity, ${ }^{10,11}$ and the ability to be produced from renewable feedstocks. ${ }^{12}$ Ron and Rosenberg ${ }^{13}$ classified biosurfactants into high molecular weight and low molecular weight molecules. The glycolipids, such as rhamnolipids, sophorolipids, and trehalolipids, are the best known and most extensively studied of the lower molecular weight biosurfactants.

In recent years biosurfactants have attracted increasing interest as potential substitutes for conventional synthetic surfactants, especially for applications such as bioremediation and microbial

\section{Received: $\quad$ May 5, 2011}

Revised: June 1, 2011

Published: June 09, 2011 


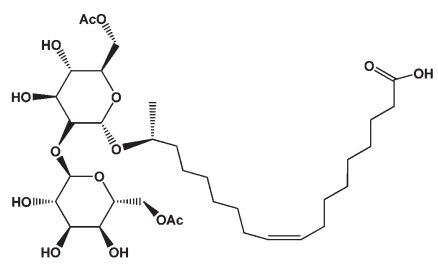

(a) Acidic sophorolipid (AS)

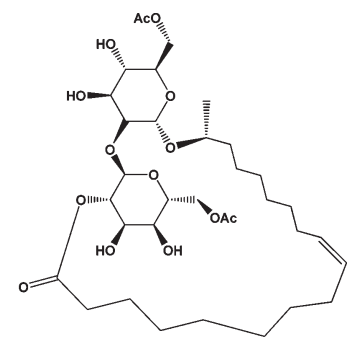

(b) Lactonic sophorolipid (LS)

Figure 1. Chemical structure of the acidic (AS) and the lactonic sophorolipids (LS).

enhanced oil recovery. ${ }^{14,15}$ However, their wider applications have been limited by the problems of large scale production and the relatively high cost, the difficulties encountered in obtaining pure biosurfactant components, and the lack of understanding of the fundamental physicochemical properties of biosurfactants. Despite considerable progress made in studying the production of biosurfactants, very few papers have reported the surface and solution behavior of biosurfactant and biosurfactant/surfactant mixtures in any detail.

Potential applications in a wider area of product formulations are likely to initially use blends of biosurfactants with conventional surfactants, and hence a fundamental understanding of their adsorption properties is essential. Recently we have used NR to study the adsorption behavior of a different biosurfactant, the rhamnolipids $\mathrm{R} 1$ and $\mathrm{R} 2$, and their mixtures with a conventional anionic surfactant, sodium 6-dodecyl benzene sulfonate, LAS. ${ }^{16,17}$ Here we have used a similar approach to study the adsorption of a different glycolipid with a fundamentally different structure, and its coadsorption with LAS. The motivation of the study was to quantify and understand how the dramatic changes in the glycolipid structure impacts upon the adsorption properties.

The hydrophobic moiety is predominantly hydroxy-oleic acid, and the sophorose headgroup is a glucose disaccharide. There are two main structural variations in the sophorolipids: ${ }^{18}$ the nature of the hydrophobic moiety (lactone or free acid form) and the extent of esterification. The two dominant sophorolipid structures are shown in Figure 1.

There is a limited amount of published data on the surface activity of sophorolipids, and we review briefly some of that data that are particularly relevant to this work. Most of the published papers have focused on the lactonic sophorolipids, because it is the most abundant form produced by Candida bombicola. ${ }^{19-21}$ However the exact composition can vary with the type of hydrocarbon substrate used in sophorolipid production and on the production conditions. ${ }^{18,19}$ Solaiman et al. ${ }^{21}$ reported the CMC and limiting surface tension values for sophorolipid mixtures containing $81.6 \mathrm{~mol} \%$ of diacetylated C18:1 LS (alkyl chain of 18 carbon and 1 double bond). They quoted limiting surface tension values of $37 \mathrm{mN} \mathrm{m}^{-1}$ at $\mathrm{pH} 6$ and $38 \mathrm{mN} \mathrm{m}^{-1}$ at $\mathrm{pH} 9$, and CMC values of $0.9 \times 10^{-5} \mathrm{M}$ at $\mathrm{pH} 6$ and $1.9 \times 10^{-5} \mathrm{M}$ at $\mathrm{pH} 9$, respectively. Otto et $\mathrm{al}^{22}$ purified sophorolipids by silica gel chromatography to obtain pure diacetylated C18:1 LS, and measured the limiting surface tension at $\mathrm{pH}$ 7.4. They reported a CMC value of $3 \times 10^{-5} \mathrm{M}$ and a limiting surface tension value of $36 \mathrm{mN} \mathrm{m}^{-1}$ at the CMC. They also found that the purified sophorolipids exhibited a higher surface activity than the crude sophorolipid mixtures, which were quoted to have a limiting surface tension of $39 \mathrm{mN} \mathrm{m}^{-1}$ at the CMC and a CMC value of
Table 1. Sample Composition of Four Different h-AS Samples by HPLC-MS

\begin{tabular}{lcccc} 
& diacetylated AS & monoacetylated AS & nonacetylated AS & LS \\
S1 & $89.4 \%$ & $2.2 \%$ & 0 & $8.4 \%$ \\
S2 & $13.6 \%$ & $66.5 \%$ & $7.9 \%$ & $12.0 \%$ \\
S3 & 0 & 0 & $93.2 \%$ & $6.8 \%$ \\
S4 & $8.5 \%$ & $39.0 \%$ & $52.5 \%$ & 0 \\
\hline
\end{tabular}

$9.5 \times 10^{-4} \mathrm{M}$. Daverey et al measured the surface tension of sophorolipid mixtures consisting primarily of diacetylated C18:1 $\mathrm{LS}$, and they reported a limiting surface tension of $34 \mathrm{mN} \mathrm{m}^{-1}$ at the $\mathrm{CMC}$ and a $\mathrm{CMC}$ value of $8.3 \times 10^{-5} \mathrm{M}$.

Zhang et al. ${ }^{23}$ investigated the effect of alkyl ester chain length on the surfactant CMC, and they found that the CMC for sophorolipid esters decreases by $50 \%$ for each additional $\mathrm{CH}_{2}$ group attached to the carboxyl end of the alkyl ester. The extent of esterification, either acetylation or lactonization also determines the solubility of the final product. Otto et al. ${ }^{22}$ reported that crude sophorolipid mixtures had a water solubility of 2$3 \mathrm{~g} \mathrm{~L}^{-1}$. In contrast, the purified diacetylated C18:1 LS is much less water-soluble (with a maximum solubility of $70 \mathrm{mg} \mathrm{L}^{-1}$ ). They also investigated the impact of the level of acetylation on the surface behavior of sophorolipids. The monoacetylated C18:1 LS is less surface active and has a higher surface tension of $40 \mathrm{mN} \mathrm{m}^{-1}$ at the $\mathrm{CMC}$ than the diacetylated form.

\section{EXPERIMENTAL DETAILS}

(a). Materials. The hydrogeneneous sophorolipids were purchased from Soliance Co. (France), and were separated into pure lactonic (LS) and acidic sophorolipid (AS) using MPLC. A chloroform/methanol (98:2 to $96: 4, \mathrm{v} / \mathrm{v}$ ) solution was used to elute the major lactonic sophorolipids from the column. This was followed by a $80: 20$ and $50: 50 \mathrm{v}: \mathrm{v}$ mixture of chloroform: methanol to elute the acidic sophorolipids. For the deuterated sophorolipids a Candida bombicola strain was grown in culture, fed with $\mathrm{D}_{2} \mathrm{O}$ and perdeuterated isostearic acid. The production and initial extraction method is described in detail elsewhere. ${ }^{24}$ The same separation procedures were used for both the hydrogenous and deuterated sophorolipids. The TLC of the sophorolipid mixture revealed the difference in polarity for LS and AS. In chloroform/ methanol $(85 / 15)$ as the mobile phase the major acidic sophorolipids had an $R_{\mathrm{f}}$ (retention factor) value of 0.47 and the major lactonic sophorolipids had an $R_{\mathrm{f}}$ value of 0.60 . Analysis by HPLCMS was used to confirm the separation. However, it was not possible to obtain pure h-AS (with 2 acetyl groups) without the presence of a trace amount of h-LS (with no acetyl groups). A number of separation techniques including MPLC, prep-TLC, prep-HPLC, and reversed phase column chromatography were attempted but the h-AS sample (with 2 acetyl groups) always contained a very small amount of h-LS (with no acetyl groups). There are four different types of h-AS samples. For clarity, the detailed composition information of a range of h-AS samples is listed in Table 1.

Compared with the rhamnolipids, ${ }^{16}$ the HPLC-MS analysis revealed a much more complicated mixture for each of the sophorolipids. The possible structural variations include length of fatty acid chain (C16-22), number of double bonds on the chain $(0-2)$, and number of acetyl groups attached to the sophorose sugar headgroup $(0-2)$. For the separated h-LS, 13 different 


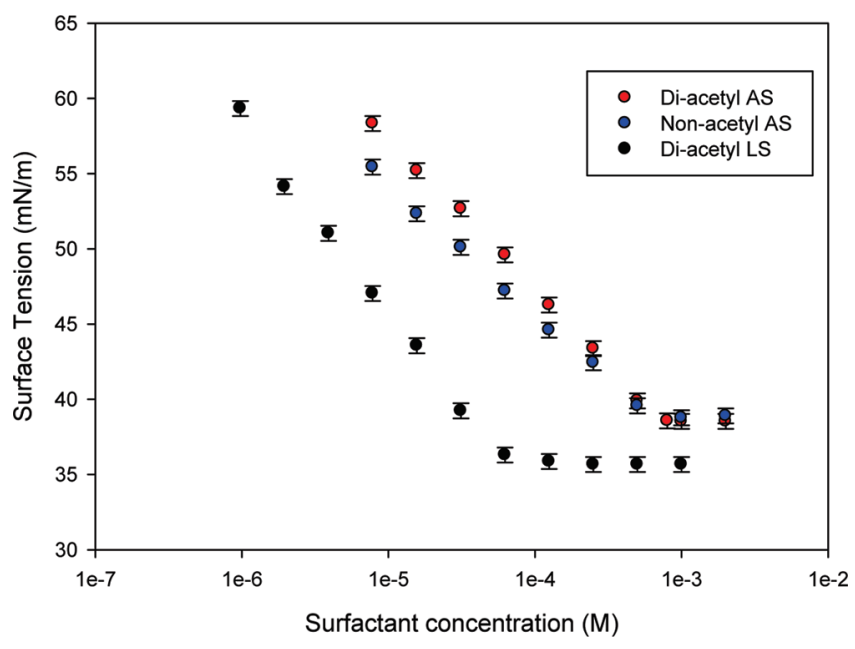

Figure 2. Surface tension data for diacetyl LS, diacetyl AS, and nonacetyl AS.

homologues were identified, among these, $87 \%$ (mole ratio) were LS with 2 acetyl groups and $13 \%$ were LS with 1 acetyl group. The most abundant structure is C18:1 ( 1 double bond) with 2 acetyl groups, and this accounts for $54 \%$ of all h-LS mixtures.

For deuterated LS, only the C18:1 (2 acetyl) structure was produced in deuterated form, but the number of hydrogen atoms that are deuterated varied from 34 to 38. In the deuterated LS sample, $77 \%$ were C18:1 (2 acetyl) structures, which can be subdivided into $54 \%$ deuterated and $23 \%$ hydrogenated. For the deuterated AS, only the C18:1 (2 acetyl) structure was in deuterated form with the number of deuterium molecules in the structure varying from 34 to 38 . The most abundant AS structure is C18:1 (2 acetyl), which accounts for $85 \%$ of the total, of which $59 \%$ is d-AS, $26 \%$ is h-AS. A further $15 \%$ are h-AS of different structures, which vary in chain length, number of double bond and number of acetyl group.

The perdeuterated isostearic acid used to produce the deuterated sophorolipids was made by exchange of the hydrogen in isostearic acid with deuterium from $\mathrm{D}_{2} \mathrm{O}$ in the presence of sodium peroxide and platinum oxide catalyst (Adam's catalyst). The sodium 6-dodecyl benzene-4 sulfate, LAS, (6-phenyl isomer) was used in two forms, with and without the dodecyl chains and phenyl ring deuterium labeled (d-LAS and h-LAS). The preparation, purification and characterization of the LAS are described in detail elsewhere. ${ }^{25}$

The aqueous solutions of the sophorolipid/LAS mixtures were prepared by weight in UHQ water (for the surface tension measurements) and in null reflecting water, NRW (a 92 vol \% $\mathrm{H}_{2} \mathrm{O} / 8$ vol \% $\mathrm{D}_{2} \mathrm{O}$ mixture), for the NR measurements or in $\mathrm{D}_{2} \mathrm{O}$ (structural NR measurements).

(b). Measurement Techniques. (i). Surface Tension. The surface tension measurements were made using a Kruss K10 maximum pull tensiometer with a $\mathrm{Pt} / \mathrm{Ir}$ du Nouy ring. The tensiometer was calibrated by the measurements in pure water before each set of measurements. The measurements were carried out at $30{ }^{\circ} \mathrm{C}$, and were made after dipping the $\mathrm{Pt} / \mathrm{Ir}$ ring in the solution and keeping it at the surface for $15 \mathrm{~min}$ to establish equilibrium conditions. The average of three repeated measurements was taken, and the experimental deviation was on the order of $\pm 0.5 \mathrm{mN} \mathrm{m}^{-1}$. The Pt/Ir ring and all associated glassware were
Table 2. Summary of Surface Tension Analysis for Lactonic and Acidic Sophorolipids

\begin{tabular}{|c|c|c|c|c|}
\hline & $\begin{array}{l}\text { surface excess, } \Gamma \\
\left( \pm 0.1 \times 10^{-10}\right. \\
\left.\mathrm{mol} \mathrm{cm}^{-2}\right)\end{array}$ & $\begin{array}{l}\text { area/molecule } \\
\AA^{2}( \pm 8)\end{array}$ & $\begin{array}{c}\text { surface tension } \\
\qquad \gamma \mathrm{mN} / \mathrm{m} \\
( \pm 0.5)\end{array}$ & $\begin{array}{c}\mathrm{CMC} / \mathrm{mM} \\
( \pm 0.02)\end{array}$ \\
\hline diacetyl LS & 2.3 & 73 & 36 & 0.06 \\
\hline diacetyl AS & 1.8 & 92 & 38.5 & 0.67 \\
\hline nonacetyl AS & 1.6 & 104 & 39 & 0.62 \\
\hline
\end{tabular}

washed in chromic acid, deionized water, and acetone, and the $\mathrm{Pt} / \mathrm{Ir}$ ring was flamed immediately before use.

(ii). Neutron Reflectivity. The neutron reflectivity measurements were made on the SURF reflectometer ${ }^{26}$ at the ISIS pulsed neutron source, UK and on the FIGARO reflectometer ${ }^{27}$ at the ILL neutron facility, France. On SURF, the measurements were made using a single detector at a fixed angle, $\theta$, of $1.5^{\circ}$, and for neutron wavelengths, $\lambda$, in the range 0.5 to $6.8 \AA$, to provide a wave vector transfer, $Q,(Q=(4 \pi / \lambda) \sin \theta, \theta$ is the grazing angle of incidence) range of 0.048 to $0.5 \AA^{-1}$. On FIGARO, a fixed angle of $3.8^{\circ}$ and neutron wavelengths between 2 and $30 \AA$ were used to provide a $Q$ range from $\sim 0.03$ to $\sim 0.25 \AA^{-1}$. The absolute reflectivity is calibrated with respect to the reflectivity of $\mathrm{D}_{2} \mathrm{O}$, and the background determined from the reflectivity at the limit of high $Q_{i}$ using well-established experimental procedures ${ }^{28}$ on SURF. On FIGARO an area detector was used to subtract the background from the specular signal, and the data were normalized to the direct beam and calibrated against $\mathrm{D}_{2} \mathrm{O}$.

In the kinematic approximation ${ }^{29}$ the specular reflectivity is related to the square of the Fourier transform of the scattering length density profile, $\rho(z)$

$$
R(Q)=\frac{16 \pi^{2}}{Q^{2}}\left|\int \rho(z) \mathrm{e}^{-i Q z} \mathrm{~d} z\right|^{2}
$$

where $\rho(z)=\sum_{i} n_{i}(z) b_{i}, n_{i}(z)$ is the number density of the $i$ th nucleus at a distance of $z$ from the interface, and $b_{i}$ is its scattering length. Application of this technique to the study of surfactant adsorption relies on the ability to manipulate $\rho(z)$ by hydrogen/ deuterium $(\mathrm{H} / \mathrm{D})$ isotopic substitution, and this has been extensively exploited at the air-water interface for a range of surfactants, and mixed surfactants ${ }^{29}$ Analysis of the reflectivity data proceeds using eq 1 or the optical matrix method ${ }^{29}$ to calculate the reflectivity from appropriate models.

\section{RESULTS AND DISCUSSION}

(i). Surface Tension. Surface tension measurements were made for the diacetylated LS, diacetylated AS and nonacetylated AS in UHQ water, and the surface tension data are shown in Figure 2. The CMC values (obtained from the intersection of straight line fits to the surface tension data below and above the $\mathrm{CMC}$ ), surface tension at the CMC, and estimates of the adsorbed amount (obtained from the slope of the $\gamma v \ln C$ plots using the Gibbs equation with a prefactor of 1 ) are summarized in Table 2 .

The diacetylated LS is more surface active than both the diacetylated AS and the nonacetylated AS. The LS has a lower surface tension at the $\mathrm{CMC}$ and the $\mathrm{CMC}$ is more than 1 order of magnitude lower than both AS samples. For LS, the surface tension results are in broad agreement with the literature data. ${ }^{20,22}$ 
Table 3. Summary of Surface Tension Analysis for AS, LS, and AS/LAS

\begin{tabular}{lcc} 
& surface tension $\gamma( \pm 0.5 \mathrm{mN} / \mathrm{m})$ & $\mathrm{CMC}( \pm 0.2 \mathrm{mM})$ \\
AS & 39 & 0.62 \\
AS LAS 70:30 & 37 & 0.53 \\
AS LAS 50:50 & 35 & 0.66 \\
AS LAS 30:70 & 36 & 0.68 \\
LAS & 34 & 1.6 \\
\hline
\end{tabular}

However, for the AS samples it is not possible to make direct comparisons with literature data, since to our knowledge there are no reported data on the surface tension of the pure acidic sophorolipids. The surface tension results presented here for both of the AS samples are, nevertheless, broadly within the surface tension range reported for sophorolipid mixtures. ${ }^{21,22}$ For the acidic sophorolipids, the diacetylated AS adsorbs slightly more strongly than the nonacetylated AS at the air/water interface but has a slightly higher CMC value. The effect of acetylation on the surface activity of acidic sophorolipids is therefore not straightforward. Otto et a. ${ }^{22}$ reported that the diacetylated LS is more effective in reducing the surface tension than monoacetylated, as previously discussed. Clenns and Cooper ${ }^{19}$ reported that the effect of lactonization on the enhancement of the surface activity is more significant than the degree of acetylation, such that the lactonic sophorolipids are more surface active than the acidic sophorolipids. However the di- and monoacetylated sophorolipids are only slightly more effective at reducing surface tension than the nonacetylated sophorolipids. ${ }^{19}$ Compared with the surface tension results previously reported for the rhamnolipids, ${ }^{16}$ the surface tension results suggest both LS and AS are less surface active than the rhamnolipids, having both a higher surface tension at the CMC and a higher CMC.

The surface tension has also been measured for LS/AS, LS/ LAS, and AS/LAS mixtures (at solution compositions of 30/70, $50 / 50$, and $70 / 30 \mathrm{~mol}$ ratios). The surface tension data for LS/LAS are shown in Figure 1 in the Supporting Information, and the key parameters obtained from the surface tension curves are summarized in Table 1 in the Supporting Information. The variation in CMC with solution composition is shown in Figure 2 in the Supporting Information.

Similar ST data were obtained for the AS/LS sophorolipid mixture (see Figure 3 and Table 2 in the Supporting Information). For both the LS/LAS and AS/LS mixtures, the ST data are consistent with ideal mixing.

For the AS/LAS mixture the mixing behavior is different and the mixed CMC values at the compositions measured are systematically lower than that predicted for ideal mixing (see Table 3 and Figure 3).

Here the AS/LAS mixture shows a departure from ideal mixing and, as illustrated in Figure 3, is consistent with a nonideality interaction parameter, $\beta$, of -2.0 , calculated from regular solution theory, RST.

The LS are weakly charged in UHQ water, whereas the headgroups of LAS are negatively charged in water, and it would be expected that nonideal mixing would occur in the LS/LAS mixture, as observed in most nonionic/ionic mixtures. ${ }^{25,30-34}$ Hines et al. ${ }^{34}$ reported ideal surfactant mixing for $n$-dodecyl- $\beta$-Dmaltoside $\left(\mathrm{C}_{12}\right.$ maltoside $)$ and $n$-dodecyl- $N, N^{\prime}$-dimethylamino betaine ( $\mathrm{C}_{12}$ betaine $) . \mathrm{C}_{12}$ maltoside is a nonionic surfactant and $\mathrm{C}_{12}$ betaine is a zwitterionic surfactant, which is electrically neutral

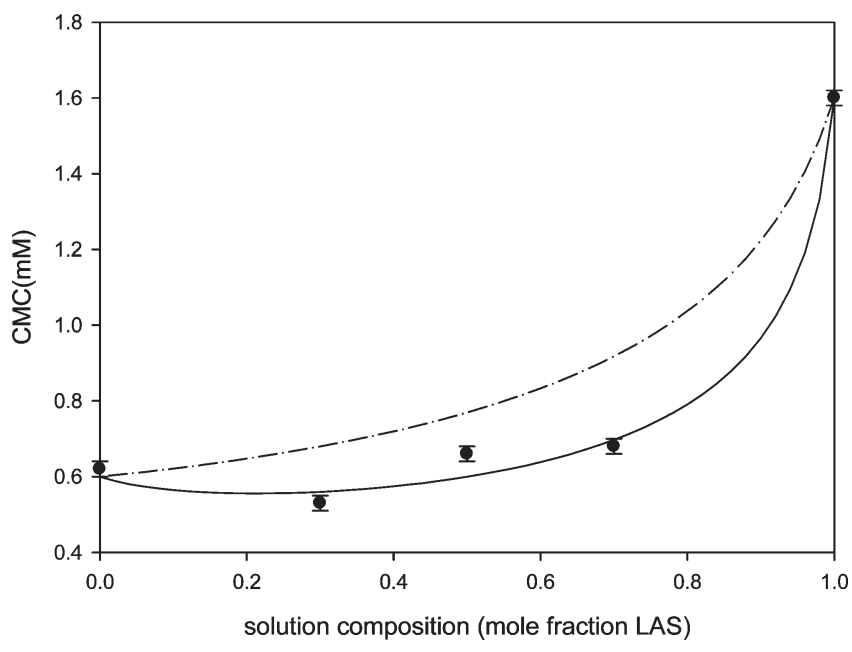

Figure 3. Variation in CMC with solution composition for AS/LAS mixtures. The dashed line is the mixed CMC for ideal surfactant mixing, and the solid line is an RST calculation for nonideal mixing with a nonideality parameter, $\beta$, of -2.0 .

at $\mathrm{pH} 7$, so there is no net electrostatic interaction. In that case the ideal mixing observed was attributed to the two surfactants having the similar headgroup areas. Hines et al also discussed nonideal mixing of SDS and $\mathrm{C}_{12}$ maltoside. Like LAS, the headgroup of SDS is negatively charged in water and the electrostatic interaction was assumed to be the main source contributing to the enthalpic interaction resulting in nonideal mixing. Nonideal mixing, consistent with RST, was also reported for the anionic/ nonionic surfactant mixtures of SDS $/ \mathrm{C}_{12} \mathrm{E}_{6}$ in micelles and at the air-water interface. ${ }^{30,31}$ In the absence of electrolyte typical values of $\beta \approx-5.0$ to -7.0 are reported, whereas in the presence of electrolyte $\beta$ is typically $\sim-2.0$ to -3.0 . Hence the observation of ideal mixing suggests that the electrostatic interaction between the LS and LAS molecules at the surface must be relatively weak, or partially mediated by the dominance of the greater surface activity of the LS component. The ideal mixing for the AS/LS mixture further indicates that the AS and LS sophorolipid components are only weakly ionic at neutral $\mathrm{pH}$, and behave broadly like nonionic surfactants. The notable exception in the ST behavior of the binary mixtures involving the sophorolipids is the AS/LAS mixture, where nonideal mixing is observed, and the interaction parameter, $\beta, \sim-2.0$, is consistent with a relatively weak interaction between the two surfactants.

(ii). Neutron Reflectivity. Neutron reflectivity measurements at the air-water, A/W, interface have been made for the LS and AS components, the AS/LS, AS/LAS, and LS/LAS binary mixtures, and the AS/LS/LAS ternary mixtures. These measurements provide a direct measure of the adsorbed amounts, composition, and structure at the surface, which extend substantially the information obtained from the ST data especially for the mixtures of surfactants.

(a). AS and LS Adsorption. Neutron reflectivity measurements were made at the air-solution interface for the deuterated surfactant (d-LS and d-AS) in NRW at surfactant concentrations from well below the CMC to above the CMC. Under these conditions the reflected signal arises only from the adsorbed layer of deuterated surfactant at the interface. Figure 4 shows some of the reflectivity data for d-LS in NRW at surfactant concentrations of $1 \times 10^{-3}, 1 \times 10^{-5}$, and $5 \times 10^{-6} \mathrm{M}$. In the Q range measured 


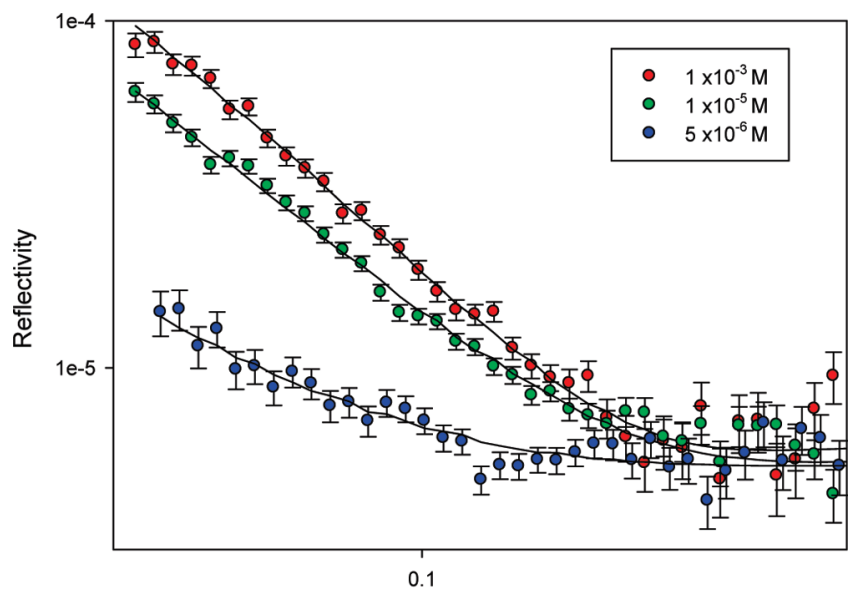

Wavevector Transfer, $Q\left(\AA^{-1}\right)$

Figure 4. Neutron reflectivity profiles for d-LS in NRW at different surfactant concentrations. For clarity the data for $5 \times 10^{-4}, 1 \times 10^{-4}$, $5 \times 10^{-5}$, and $5 \times 10^{-7} \mathrm{M}$ (see Table 4$)$ are not shown. The solid lines are model calculations as described in the text for the parameters listed in Table 4.

the absolute level of the reflectivity gives a measure of the adsorbed amount, which decreases with decreasing surfactant concentration, and the slope is related to the thickness of the adsorbed layer. Hence the reflected signal can be analyzed in terms of the adsorbed amount at the interface and the thickness of the adsorbed layer. The most direct way of determining the adsorption is to assume that the adsorbed layer can be described as a single layer of uniform composition, and this is a good description of the data presented here. The measured reflectivity is then modeled by comparing it with a calculated profile (assessed by least-squares criterion), using the optical matrix method, ${ }^{35}$ for this simple structural model. The model parameters are the scattering length density, $\rho$, and the thickness, $\tau$, of the adsorbed layer, and are related to the adsorbed amount or area/molecule, $A$, by ${ }^{29}$

$$
\tau \rho=\frac{\sum b}{A}
$$

where $\Sigma b$ is the sum of scattering lengths of the deuterated surfactant, the adsorbed amount is given by $\Gamma=1 /\left(N_{\mathrm{a}} A\right)$, and $N_{\mathrm{a}}$ is Avogadro's number.

The solid lines in Figure 4 are model calculations as described above for the parameters in Table 4, and using the scattering lengths tabulated in Table 3 in the Supporting Information, and specifically here for a scattering length of $2.86 \times 10^{-3}$ A for d-LS.

At surfactant concentrations of $5 \times 10^{-5}$ and greater (at or above the CMC), the data are consistent with a surface adsorbed amount of $2.3 \times 10^{-10} \mathrm{~mol} \mathrm{~cm}{ }^{-2}$, in good agreement with the value obtained from the surface tension. Hence, in the evaluation of the surface tension data, the Gibbs prefactor was correctly assumed to be unity, and this indicates that the LS component is only weakly ionic, similar to what was recently reported for the rhamnolipids. ${ }^{16}$

Typical reflectivity data for d-AS in nrw at surfactant concentrations of $2 \times 10^{-3}, 5 \times 10^{-4}, 1 \times 10^{-4}, 1 \times 10^{-5}$, and $5 \times 10^{-6} \mathrm{M}$ are shown in Figure 4 in the Supporting Information, and the corresponding model parameters are listed in Table 4 in the Supporting Information. The CMC of diacetyl-AS is $\sim 6.7 \times 10^{-4} \mathrm{M}$, and the adsorbed amount of diacetyl-AS is $1.8 \times 10^{-10} \mathrm{~mol} \mathrm{~cm}^{-2}$
Table 4. Model Parameters from Analysis of Neutron Reflectivity Data for d-LS in NRW

\begin{tabular}{ccccc}
$\begin{array}{c}\text { surfactant } \\
\text { concentration } \\
(\mathrm{M})\end{array}$ & $\begin{array}{c}\tau / \AA \\
( \pm 2)\end{array}$ & $\begin{array}{c}\rho \\
\left( \pm 0.2 \times 10^{-6} \AA^{-2}\right)\end{array}$ & $\begin{array}{c}A \\
\left(\AA^{2}\right)\end{array}$ & $\begin{array}{c}\Gamma \\
\left( \pm 0.1 \times 10^{-10}\right. \\
\left.\mathrm{mol} \mathrm{cm}^{-2}\right)\end{array}$ \\
$1 \times 10^{-3}$ & 25 & 1.6 & $72 \pm 2$ & 2.3 \\
$5 \times 10^{-4}$ & 21 & 1.8 & $78 \pm 2$ & 2.1 \\
$10^{-4}$ & 25 & 1.6 & $71 \pm 2$ & 2.3 \\
$5 \times 10^{-5}$ & 21 & 1.9 & $74 \pm 2$ & 2.3 \\
$1 \times 10^{-5}$ & 26 & 1.1 & $98 \pm 4$ & 1.7 \\
$5 \times 10^{-6}$ & 31 & 0.5 & $203 \pm 10$ & 0.8 \\
$5 \times 10^{-7}$ & 31 & 0.2 & $406 \pm 10$ & 0.4 \\
\hline
\end{tabular}

above the CMC, at solution concentrations of $1 \times 10^{-3}$ and $2 \times$ $10^{-3} \mathrm{M}$. The surface tension results are again in good agreement with the NR data with a Gibbs prefactor of 1.0, and this also indicates that the AS component is only weakly ionic. However, at solution concentrations below the CMC at $5 \times 10^{-4}$ and $0.1 \times$ $10^{-4} \mathrm{M}$, the NR results show a slight initial increase in surfactant adsorbed amount with decreasing surfactant concentration. This trend continues until the concentration is reduced to $1 \times 10^{-4} \mathrm{M}$, and the adsorbed amount decreases with further decreases in the surfactant concentration. This initial increase is unexpected, and must be caused by the presence of trace amount of surface active impurities in the deuterated diacetyl-AS sample, probably other surface active AS isomers. Impurities would normally result in a minimum in the surface tension data in the region of the CMC, and this is not observed in the corresponding ST data.

The adsorption isotherms for the LS and AS sophorolipids have a concentration dependence that is consistent with a Langmuir isotherm of the form

$$
\Gamma=\frac{\Gamma_{\max } C}{C+k}
$$

where $\Gamma$ and $\Gamma_{\max }$ are the adsorbed amounts and the maximum adsorption, $C$ is the surfactant concentration, and $k$ is the adsorption coefficient. The resulting adsorption isotherms for LS and AS are plotted in Figure 5, panels a and b.

The parameters from the Langmuir fits show that AS and LS have similar adsorption coefficient, $k$, value, indicating that both sophorolipids have similar affinities for the air-water interface. AS has a smaller adsorbed amount, $1.94 \times 10^{-10} \mathrm{~mol} \mathrm{~cm}^{-2}$ compared with $2.28 \times 10^{-10} \mathrm{~mol} \mathrm{~cm}{ }^{-2}$ for the LS; that is, the LS is more surface active. In lactonic sophorolipids, the lactone is obtained by reacting the carboxyl group of the fatty acid with the $4^{\prime}$ hydroxyl group of the terminal glucose in sophorose through an esterification reaction. The increase in the hydrophobicity and hence the surface activity is associated with the loss of the carboxyl group.

(b). Binary and Ternary Mixtures of AS/LS, AS/LAS, LS/LAS, and $A S / L S / L A S$. Neutron reflectivity measurements were made for binary and ternary surfactant mixtures of AS/LS, AS/LAS, LS/LAS, and AS/LS/LAS at a concentration of $1 \mathrm{mM}$ in NRW, above the mixed CMC, and over a wide range of solution compositions. Both the LS and the AS samples (deuterated and hydrogenous) used in the NR measurements were the diacetylated versions.

(i). Binary Mixtures. For the binary surfactant mixtures, the NR measurements were made for the two complementary 
(a)

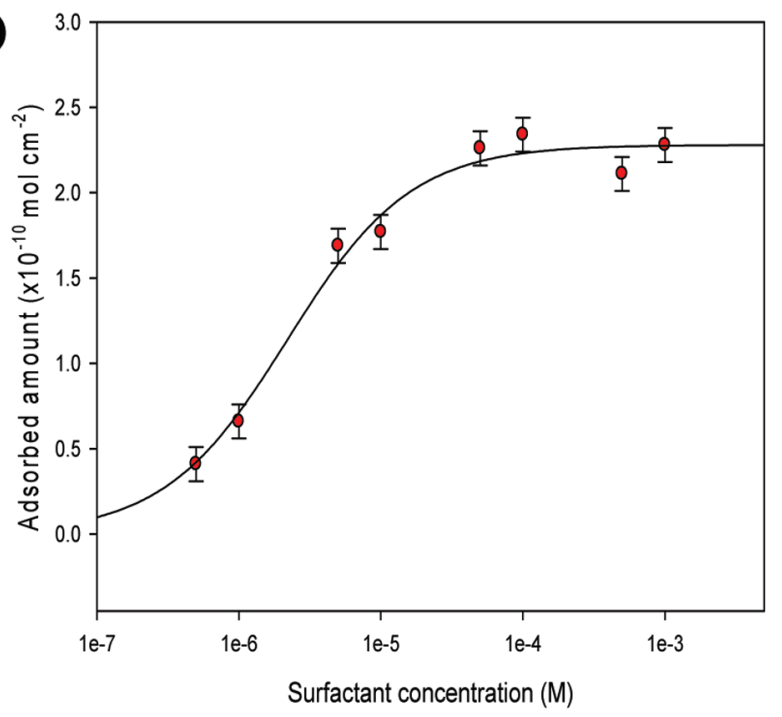

(b)

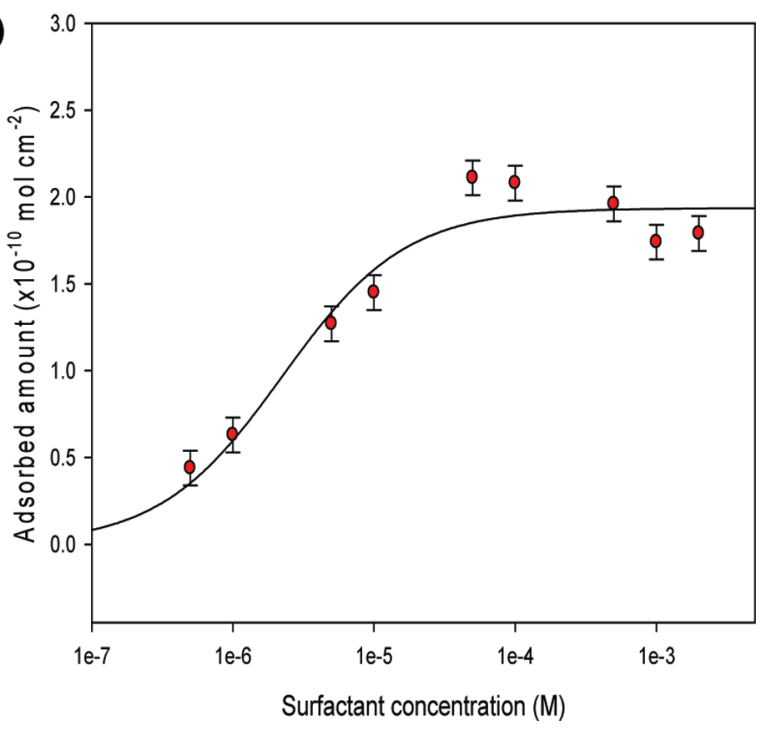

Figure 5. Adsorption isotherm for (a) LS, solid line in a fit to a Langmuir with $k=2.2 \times 10^{-6}$ and $\Gamma_{\max }=2.28 \times 10^{-10} \mathrm{~mol} \mathrm{~cm}^{-2}$, and (b) AS, solid line in a fit to a Langmuir with $k=2.2 \times 10^{-6}$ and $\Gamma_{\max }=1.94 \times 10^{-10} \mathrm{~mol} \mathrm{~cm}^{-2}$.

(a)

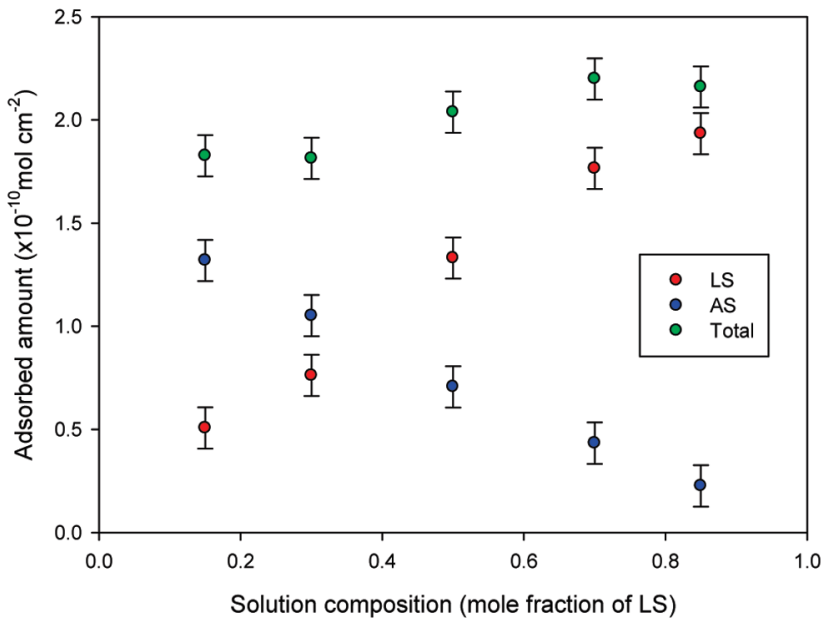

(b)

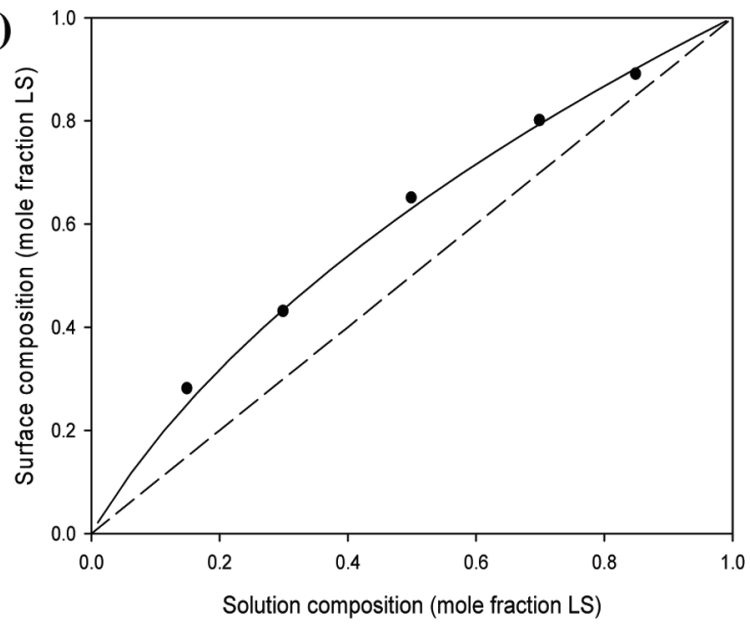

Figure 6. LS/AS mixtures at a solution concentration of $1 \mathrm{mM}$ at the air/water interface at $30{ }^{\circ} \mathrm{C}$. (a) Variation in surfactant adsorbed amount with solution composition; (b) variation in surface composition with solution composition. The dashed line is for equivalent surface and solution compositions. The solid line is an RST calculation assuming ideal mixing and for CMC values in Table 2 in the Supporting Information.

isotopic combinations, d-LS/h-AS and h-LS/d-AS in NRW. For LS/LAS mixtures a further set of measurements were made at a fixed solution composition of 50/50 mol ratio and over a range of solution concentrations from $0.2 \mathrm{mM}$ (below the mixed CMC) to $2 \mathrm{mM}$ (above the mixed CMC). The hydrogenous AS samples have a residual $8.4 \%$ mole fraction of the LS component, and this imposed some limitations on the compositions of the LS/AS mixtures that could be investigated. For AS/LAS binary mixtures, it is not possible to use the hydrogenous AS sample directly in the measurements because of the presence of the trace amounts of LS in the sample. Hence the NR measurements for the AS/LAS mixtures were made using the combination of d-AS/h-LAS and d-AS/d-LAS in NRW. The area per molecule of each surfactant component adsorbed at the $\mathrm{A} / \mathrm{W}$ interface was obtained by solving directly the two resulting simultaneous equations (see eq 4 below).
The reflectivity was evaluated using the optical matrix method, ${ }^{35}$ as described earlier, and the area per molecule of each component adsorbed at the $\mathrm{A} / \mathrm{W}$ interface was obtained by solving the associated simultaneous equations. That is, the variation in adsorbed layer composition with solution composition was determined using eq 4 for the binary mixture.

$$
\rho \tau=\sum b_{1} / A_{1}+\sum b_{2} / A_{2}
$$

where $b_{i}$ and $A_{i}$ are the scattering lengths and area/molecule of each component of the binary mixture. Equation 4 was extended to include an additional term $\Sigma b_{3} / A_{3}$ for the ternary mixtures (see later section).

For LS/AS binary mixtures at a concentration of $1 \mathrm{mM}$, the variation in adsorbed amount and surface composition with surfactant solution composition are shown in figure 6, and the 
Table 5. Variation in Adsorbed Amounts and Surface Composition for $1 \mathrm{mM} \mathrm{LS} / \mathrm{AS}$ in UHQ Water

\begin{tabular}{|c|c|c|c|c|c|c|c|}
\hline $\begin{array}{l}\text { solution composition } \\
\text { (mole fraction LS) }\end{array}$ & contrast LS/AS & $\begin{array}{c}\tau / \AA \\
( \pm 1)\end{array}$ & $\begin{array}{c}\rho \\
\left( \pm 0.2 \times 10^{-6} \AA^{-2}\right)\end{array}$ & $A / \AA^{2}$ & $\begin{array}{c}\Gamma \\
\left( \pm 0.2 \times 10^{-10} \mathrm{~mol} \mathrm{~cm}^{-2}\right)\end{array}$ & $\begin{array}{c}\Gamma_{\text {total }} 10^{-10} \mathrm{~mol} \mathrm{~cm}^{-2} \\
( \pm 0.04)\end{array}$ & $\begin{array}{c}\text { Surface composition } \\
\text { (mole fraction LS) } \pm 0.02\end{array}$ \\
\hline \multirow[t]{2}{*}{0.85} & $\mathrm{dh}$ & 26 & 1.34 & $86 \pm 4$ & 1.93 & 2.16 & 0.89 \\
\hline & hd & 28 & 0.50 & $734 \pm 20$ & 0.23 & & \\
\hline \multirow[t]{2}{*}{0.7} & $\mathrm{dh}$ & 24 & 1.39 & $94 \pm 4$ & 1.77 & 2.20 & 0.80 \\
\hline & hd & 33 & 0.52 & $383 \pm 10$ & 0.43 & & \\
\hline \multirow[t]{2}{*}{0.5} & $\mathrm{dh}$ & 27 & 1.01 & $125 \pm 4$ & 1.33 & 2.04 & 0.65 \\
\hline & hd & 32 & 0.63 & $235 \pm 10$ & 0.71 & & \\
\hline \multirow[t]{2}{*}{0.3} & dh & 30 & 0.65 & $218 \pm 10$ & 0.76 & 1.81 & 0.42 \\
\hline & hd & 29 & 1.24 & $158 \pm 4$ & 1.05 & & \\
\hline \multirow[t]{2}{*}{0.15} & dh & 36 & 0.43 & $373 \pm 10$ & 0.51 & 1.83 & 0.28 \\
\hline & hd & 27 & 1.01 & $126 \pm 4$ & 1.32 & & \\
\hline
\end{tabular}

associated parameters are summarized in table 5. The parameters from the analysis of the reflectivity data for the LS/LAS, AS/LAS, and LS/AS/LAS ternary mixtures are summarized in Tables 5-9 in the Supporting Information.

The data in Figure 6a shows that as the solution composition changes from AS rich to LS rich, the amount of LS adsorbed at the interface increases almost linearly, the adsorbed amount of AS decreases linearly, and the total adsorbed amount increases slightly. The adsorbed amounts, measured by NR, for the pure LS and AS are 2.3 and $1.8 \times 10^{-10} \mathrm{~mol} \mathrm{~cm}^{-2}$, respectively, and compared with these values, the total adsorption for LS/AS mixtures of different compositions shows no synergistic enhancement in the total adsorption. Figure $6 \mathrm{~b}$ shows the variation in surface composition of LS with its solution composition, and it is clear that the LS component dominates the surface adsorption over the entire range of compositions measured. The measurements were made at a fixed surfactant concentration of $1 \mathrm{mM}$, which is about $5-7$ times greater than the mixed CMC over most of the composition range (see Figure 3 and Table 2 in the Supporting Information). The dominance of LS at the interface is, however, still consistent with ideal mixing, and the solid line is a calculated curve for ideal mixing, based on regular solution theory (RST). ${ }^{36}$ This is also consistent with the surface tension data which also exhibited ideal mixing (see Figure 3 in the Supporting Information), and the calculated curve in Figure $6 \mathrm{~b}$ is based on the measured CMC values from Table 2 and Figure 3 in the Supporting Information. As reported previously for rhamnolipids, ${ }^{16}$ a similar mixing behavior has been observed for the $\mathrm{R} 1 / \mathrm{R} 2$ mixtures, where $\mathrm{R} 1$ dominates at the $\mathrm{A} / \mathrm{W}$ interface over the entire composition measured and at a concentration in similar excess of the mixed CMC. R1 is more surface active than $\mathrm{R} 2$, but also in that case the CMC values of R1 and R2 are much closer that those of the LS and AS sophorolipids. As a consequence the R1/R2 mixing behavior was not ideal and could not be readily explained by RST. For the R1/R2 mixtures, ${ }^{16}$ where the surfactant headgroups are of different size, and the alkyl chains have an identical structure, the surface mixing behavior was similar to that observed for the $\mathrm{C}_{12} \mathrm{EO}_{3} / \mathrm{C}_{12} \mathrm{EO}_{8}$ mixture, where the packing constraints of the larger $\mathrm{EO}_{8}$ group dominates. ${ }^{37}$ Here the LS and AS sophorolipids have identical surfactant headgroup structures but differ in the structure of the nonpolar alkyl chains. Hence in this case the surface behavior is more comparable to that reported for the nonionic surfactant mixture of $\mathrm{C}_{14} \mathrm{EO}_{6}$ and $\mathrm{C}_{10} \mathrm{EO}_{6}{ }^{28}$, where the surface structure (relative positions and extent of the different components at the interface) of the

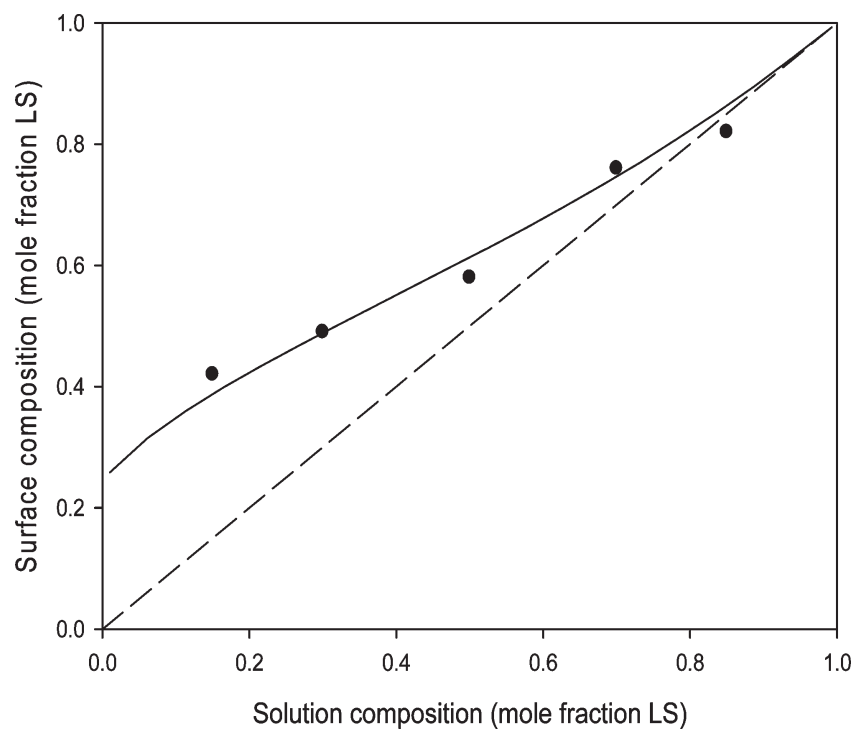

Figure 7. Variation in surface composition with solution composition, at the air/water interface at $30{ }^{\circ} \mathrm{C}$ for the LS/LAS mixture. The dashed line is for equivalent surface and solution compositions. The solid line is an RST calculation assuming ideal mixing and for CMC values in Table 5 in the Supporting Information.

mixture adjusts to accommodate the differences in alkyl chain length In order to gain further insight into the packing of the LS and AS components at the interface for the AS/LS mixture we have also measured the structure of LS/AS mixtures at a fixed composition (30/70), and this is discussed later in this paper

For the LS/LAS mixtures, the variation in adsorbed amount and surface composition with solution composition at a concentration of $1 \mathrm{mM}$ is shown in Figure 5 in the Supporting Information and Figure 7, and the key model parameters are summarized in Table 5 in the Supporting Information.

Figure 5 in the Supporting Information shows that as the surfactant solution composition varies from LAS rich to LS rich, the adsorbed amount of LS increases almost linearly, the amount of LAS adsorbed at the interface decreases almost linearly, and the total surfactant adsorption also decreases. The variation in surface composition with solution composition is shown in Figure 7. The LS component dominates over almost the entire composition measured, except at a solution composition of $85 / 15$ LS/LAS, where the surface composition is identical to the 


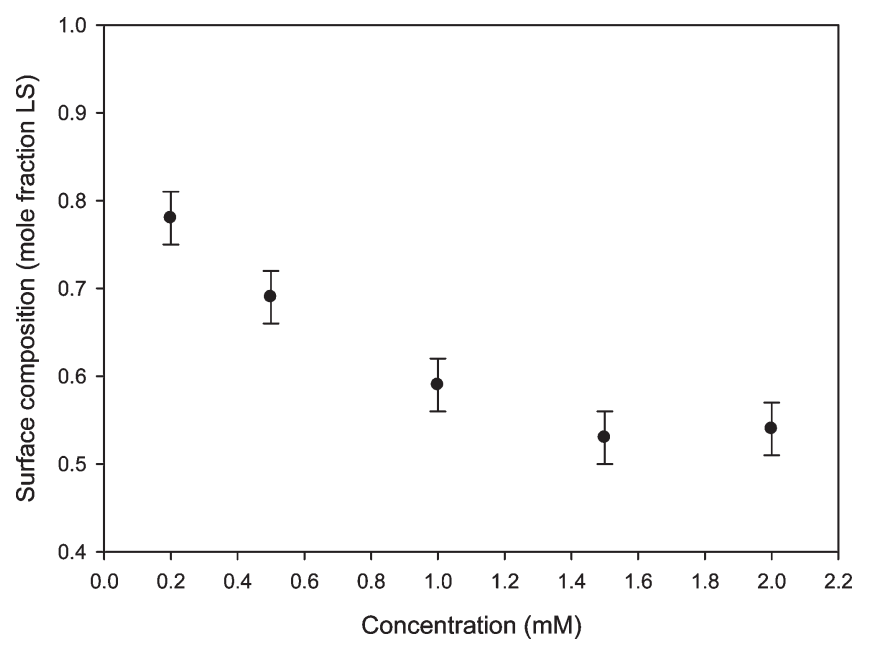

Figure 8. Variation of surface composition of LS with increasing surfactant concentration for the LS/LAS mixtures at a fixed composition $50 / 50$ mol ratio.

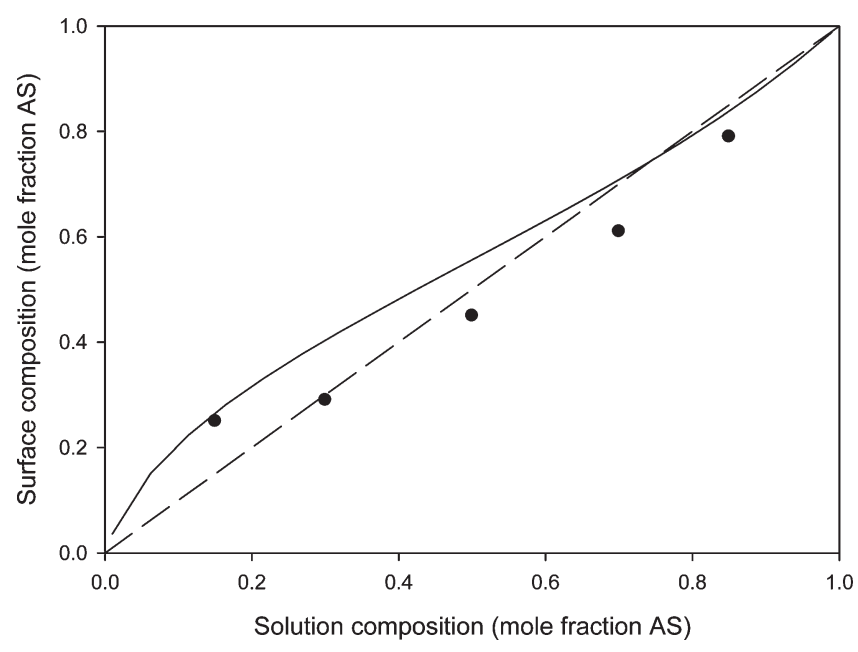

Figure 9. Variation in surface composition with solution composition, at the $\mathrm{A} / \mathrm{W}$ interface at $30^{\circ} \mathrm{C}$, for the AS/LAS mixture at a surfactant concentration of $1 \mathrm{mM}$. The dashed line is for equivalent surface and solution compositions. The solid line is an RST calculation for $\beta=-2.0$ and for the CMC values in table 3.

solution composition. Like the mixing behavior of the LS/AS mixture in Figure 6, such mixing behavior could imply a strong departure from ideal mixing, but the surface tension data are consistent with ideal mixing. However because of the large difference in the CMC values of LS and LAS reflecting the greater surface activity of LS, the concentration of the measurements relative to the mixed $\mathrm{CMC}$, and although the variation in surface composition is marked, it is still consistent with ideal mixing. This is illustrated by the solid line in Figure 7, which is an RST calculation, assuming ideal mixing and the CMC values from surface tension (see Table 1 in the Supporting Information). Penfold et al. ${ }^{25}$ measured the adsorbed amount of pure LAS in UHQ water using NR and reported a value of $3.1 \times 10^{-10}$ mol cm ${ }^{-2}$. The adsorbed amount for the pure LS is $\sim 2.3 \times$ $10^{-10} \mathrm{~mol} \mathrm{~cm}{ }^{-2}$. Comparing these values with the values of total adsorbed amounts listed in Table 5 in the Supporting Information

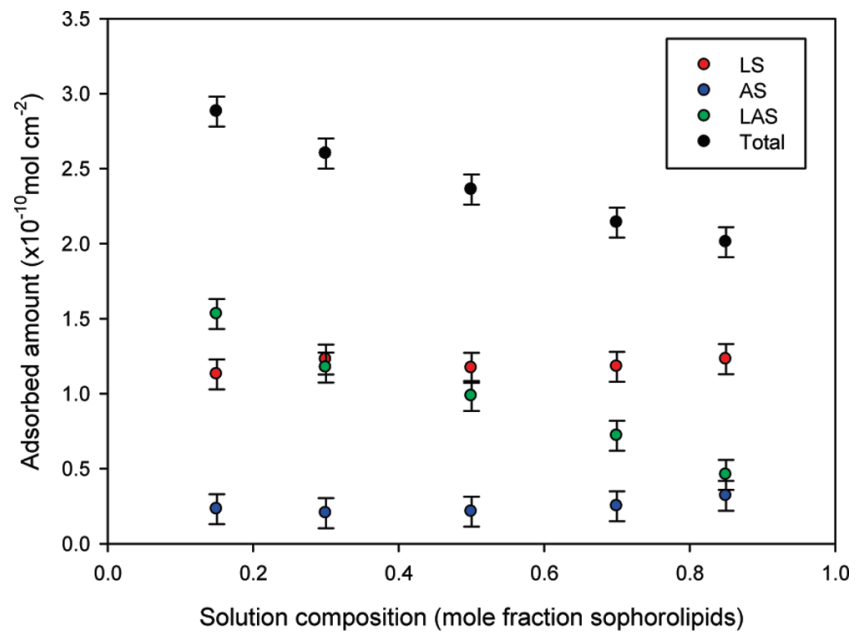

Figure 10. Variation in adsorbed amount with composition at the air/ water interface for LS:AS (2:1)/LAS; at $1 \mathrm{mM}$ solution concentration and at $30{ }^{\circ} \mathrm{C}$.

for LS/LAS mixtures, it can be concluded that there is no synergistic enhancement in the total adsorption with the LS/LAS mixtures.

In addition to the NR measurements for LS/LAS mixtures at a fixed surfactant concentration, a further set of measurements were made at a fixed solution composition (50/50 mol ratio) and at varying surfactant concentrations from $0.2 \mathrm{mM}$ (below the $\mathrm{CMC})$ to $2 \mathrm{mM}(\sim 8$ times the CMC). The adsorbed amounts are shown in Figure 6 in the Supporting Information, and figure 8 shows the variation of the surface composition of LS as a function of the surfactant solution concentration.

The variation in the adsorbed amounts (Figure 6 in the Supporting Information) and in the surface composition (Figure 8) are entirely consistent with the expectation of the pseudophase approximation and especially RST. ${ }^{36}$ That is, close to the CMC the surface is richest in the more surface active component (in this case LS) and as the concentration increases the surface evolves toward a composition closer to the solution composition, as has been previously demonstrated in conventional surfactant mixtures. ${ }^{30}$

The adsorption data for AS and LAS in AS/LAS mixtures and the variation in surface composition with solution composition are shown in Figures 7 in the Supporting Information and in Figure 9. The RST calculation for the surface composition, based on the ST CMC values and for a $\beta \approx-2.0$ is also shown as the solid line in Figure 9.

The overall trend in the adsorbed amount for AS/LAS mixtures, as shown in Figure 7 in the Supporting Information, is broadly similar to that for LS/LAS, and the total adsorption decreases for solution compositions increasingly rich in LAS. For the LS/LAS mixture the LS component dominates the surface adsorption, particularly for mixture compositions relatively rich in LAS. For the AS/LAS mixtures here the surface composition is relatively close to the solution composition over the entire composition range studied, and hence the surface mixing is close to what would be expected from ideal mixing. From the CMC variation (ST data) an interaction parameter, $\beta, \sim-2.0$ was obtained, and the corresponding variation in surface composition, calculated from RST, is shown as the solid line in figure 9. Here the more closely similar CMC values for LAS and AS result 
in similar surface activities, and hence the less pronounced variation in the surface composition than is observed for the AS/LS and LS/LAS mixtures. The RST calculation shown in figure 9 is only broadly consistent with the data, and the inadequacies in this approach must in part reflect the subtleties in the packing of the two components. This is not observed in the AS/LS and LS/LAS mixtures due to the dominance of the surface activity of LS compared to either the AS or LAS components, and the presence of an even weaker electrostatic interaction. The key parameters from the modeling of the NR data are summarized in Table 6 in the Supporting Information. Compared with the adsorbed amount reported for pure $\mathrm{AS}^{47}$ and $\mathrm{LAS}^{25}$, which are 1.8 and $3.1 \times 10^{-10} \mathrm{~mol} \mathrm{~cm}^{-2}$, respectively, the data shown in Table 6 in the Supporting Information show that there is no synergistic enhancement in the total adsorption for AS/LAS mixtures or any strongly preferential adsorption of either component to the interface.

(ii). Ternary AS/LS/LAS Mixtures. For the ternary surfactant mixtures, NR measurements were made for the isotopic combinations d-LS/h-AS/h-LAS, h-LS/d-AS/h-LAS, and h-LS/h-AS/ d-LAS in NRW and at three different LS/AS compositions (2:1, $1: 1$, and 1:2). It was possible to use the h-AS sample in these measurements (see earlier discussions) by restricting the overall range of compositions studied. The results from the neutron reflectivity measurements for the variation in adsorbed amount with solution composition for the sophorolipids (LS + AS)/LAS ternary mixtures, at three different LS/AS compositions (2:1; 1:1, and 1:2), are shown in Figure 10 and Figure 8 in the Supporting Information.

The overall trends in the adsorption are broadly similar for LS: AS (2:1)/LAS and LS:AS (1:1)/LAS mixtures; that is, as the surfactant solution composition becomes richer in sophorolipids (SL), the adsorbed amount of LS increases almost linearly, and the adsorbed amount of LAS decreases linearly. However, the adsorption of AS varies much less and the total surfactant adsorption decreases, broadly similar to the adsorption behavior of binary mixtures of LS/LAS. For the LS:AS (1:2)/LAS mixtures, the trend is quantitatively different. As the surfactant composition becomes richer in SL, the adsorbed amount of the AS initially increases up to a solution composition of 70/30, and then the amount adsorbed levels off as the solution composition of SL increases further. For the LAS component, the reverse trend is observed, and the adsorbed amount initially decreases up to a 70/30 solution composition. The total adsorbed amount remains within error constant across the entire composition range, and the variations in adsorption with solution composition are much less pronounced. Finally, the adsorbed amount of the LS varies only modestly with the increase in the amount of SL in the solution. The associated model parameters from the analysis of the NR data for 3 different mixed systems are listed in Tables 7-9 in the Supporting Information. There is no synergy in the total surfactant adsorbed amount observed for any of the three ternary mixture systems, but the total adsorbed amount is higher for LS:AS (1:2)/LAS and LS:AS (1:1)/LAS than for LS:AS (2:1)/LAS, in marked contrast to what is observed in the recently reported rhamnolipid/LAS (R1/R2/LAS) mixtures, ${ }^{26}$ where a synergistic enhancement in the total adsorption was observed for the ternary $\mathrm{R} 1 / \mathrm{R} 2 / \mathrm{LAS}$ mixtures, but not for binary mixtures of $\mathrm{R} 1 / \mathrm{R} 2$, R1/LAS, and R2/LAS. For the sophoroplipid (AS, LS)/LAS mixtures no synergistic enhancement in the adsorption is observed for the corresponding binary or ternary mixtures.

\section{(a) $2: 1 \mathrm{AS} / \mathrm{LS}$}

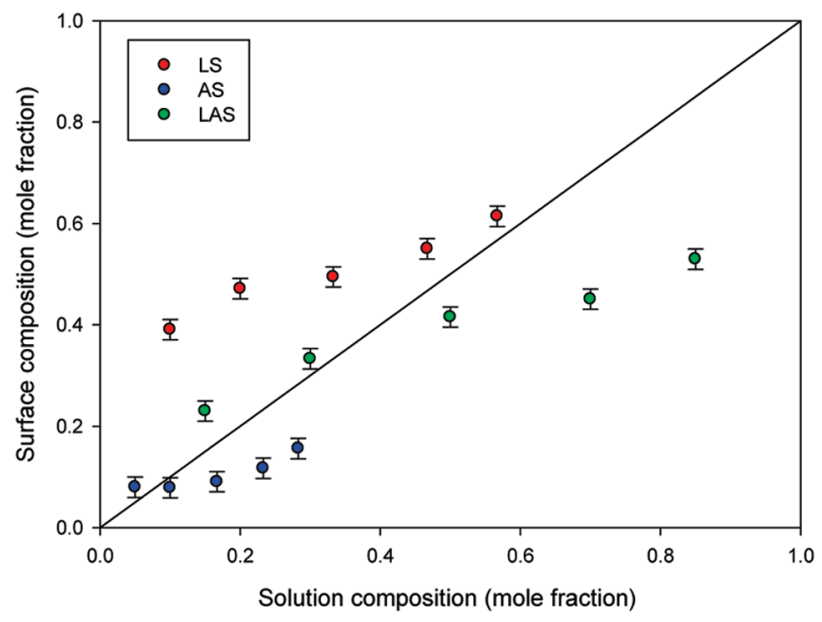

\section{(b) $1: 1 \mathrm{AS} / \mathrm{LS}$}

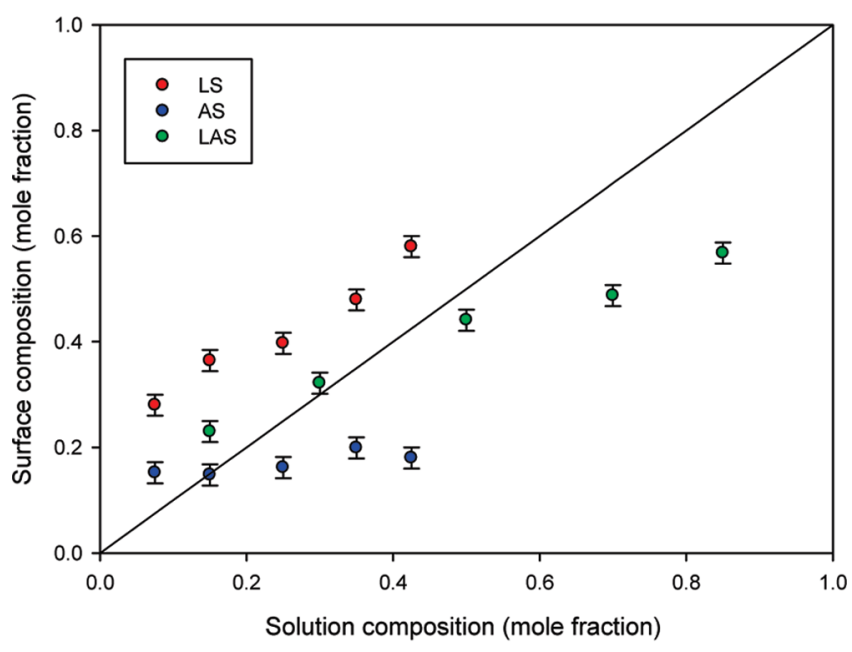

(c) $1: 2 \mathrm{AS} / \mathrm{LS}$

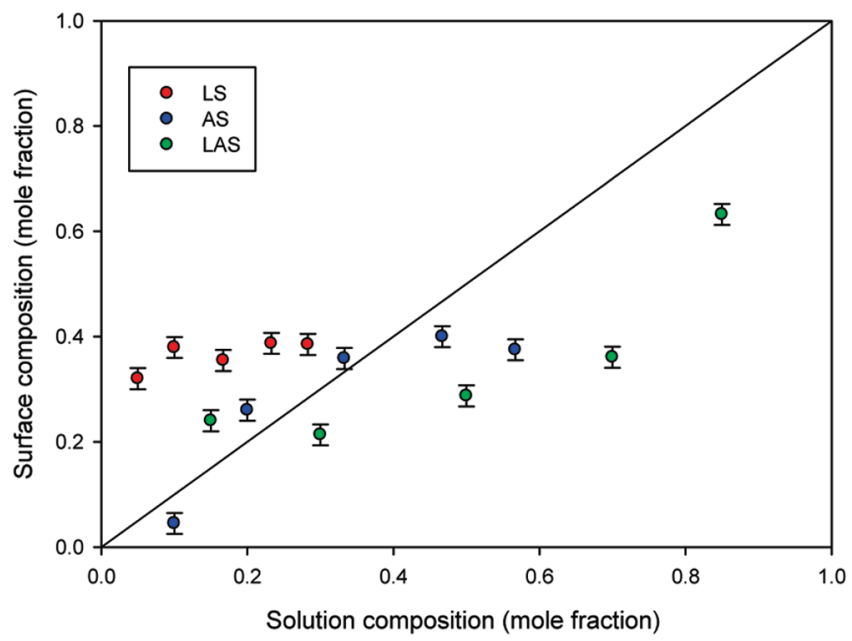

Figure 11. Variation in adsorbed amount at the $\mathrm{A} / \mathrm{W}$ interface for (a) LS:AS (2:1)/LAS, (b) LS:AS (1:1)/LAS, and (c) LS:AS (1:2)/LAS, at $1 \mathrm{mM}$ solution concentration at $30^{\circ} \mathrm{C}$. The solid line represents equal surface and solution compositions. 


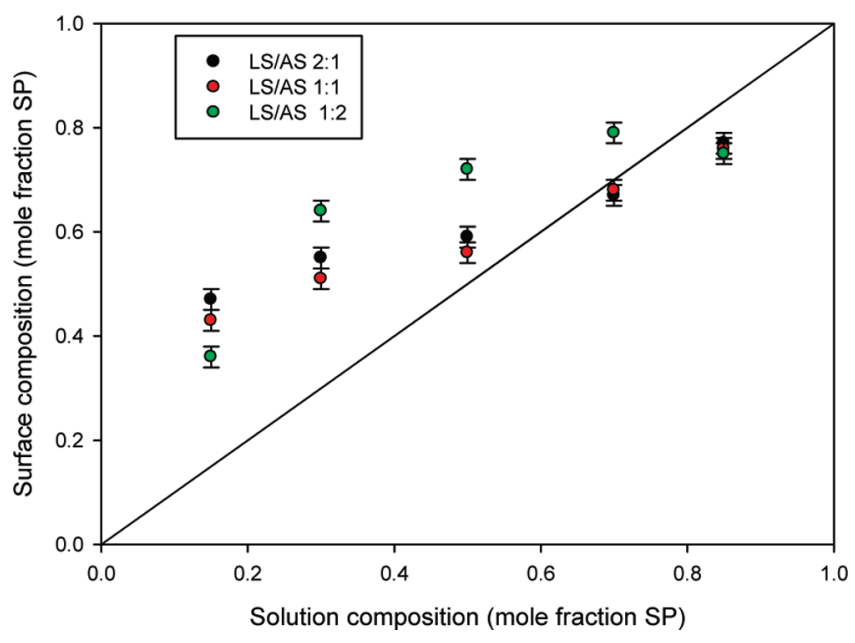

Figure 12. Variation in surface composition for the ternary LS/AS/LAS mixtures, at a surfactant concentration of $1 \mathrm{mM}$. The solid line represents equal surface and solution compositions.

In Figure $11 \mathrm{a}-\mathrm{c}$, the variation in surface composition with solution composition for the individual components of the LS/ AS/LAS ternary mixture is plotted for the three LS/AS compositions, $2: 1,1: 1$, and $1: 2$, studied.

The broad trends are again similar for LS:AS (2:1)/LAS and LS:AS (1:1)/LAS mixtures, although the details are quantitatively different. In both these mixtures, the LS component dominates the surface over the most of the composition range measured. For the LS:AS (2:1)/LAS system, the surface composition becomes closer to the solution composition as the solution becomes richer in LS. Whereas for LS:AS (1:1)/LAS, the surface composition of the LS component follows the same trend as the solution composition across the entire range of composition measured, but the surface is systematically richer in LS. In both mixtures, the surface composition of the LAS component is broadly similar to the solution composition at low solution composition, but the LAS gradually becomes depleted from the interface as the solution composition becomes increasingly rich in the sophorolipid. For the AS component, however, the surface composition shows less of a variation as the AS solution composition increases. For the LS:AS (1:2)/LAS system, the trends in the evolution of the surface composition are different again. The LS component still competes relatively favorably at the surface, but the surface composition of LS stays relatively constant as the solution composition becomes richer in sophorolipid. For AS, the surface composition is initially close to the solution composition, but the AS component becomes gradually depleted as the solution composition becomes richer in sophorolipid. Lastly, for most of the compositions measured, the surface composition of LAS is significantly lower than the solution composition, indicating that the LAS component competes least favorably for the interface. Although for the ternary mixtures (as with the binary mixtures) the LS component dominates the surface adsorption, an interesting aspect of this trend is shown in Figure 12 where the surface composition (in terms of sophorolipid, that is, AS and LS) is plotted as a function of the solution composition.

The data for 2:1 and 1:1 LS/AS ratios are broadly similar and entirely consistent with the general observations outlined earlier. However, for the LS/AS composition of 1:2, due to the larger AS adsorption, the surface is richer in sophorolipid over the entire

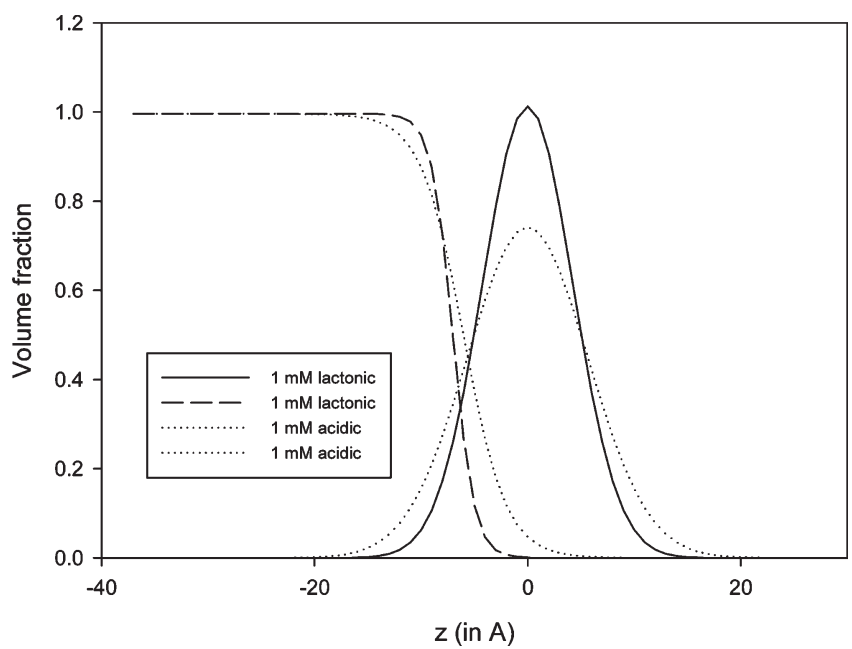

Figure 13. Volume fraction distributions for $1 \mathrm{mM}$ LS and AS at the $\mathrm{A} / \mathrm{W}$ interface, from partial structure factor analysis.

range of the solution compositions. Hence although there is no synergy in the total adsorption, for the LAS/LS/AS (1:2) mixture there is a synergy in the surface composition, strongly in favor of the sophorolipid component. This implies that at the greater solution compositions of AS, the presence of the AS component reduces the packing constraints at the surface in favor of a greater fraction of sophorolipid adsorbed at the interface.

These observations are in marked contrast to the results discussed previously for rhamnolipids ${ }^{17}$ in the ternary mixtures of three different $\mathrm{R} 1 / \mathrm{R} 2$ compositions (2:1, 1:1, and 1:2), where in that case the surface composition of LAS was consistently higher than the solution concentration. Furthermore in the binary mixtures of the rhamnolipids with LAS, the LAS adsorption largely dominated the adsorption when mixed with R2. In the ternary mixtures of LS, AS, and LAS, and also in the binary mixtures of LS/LAS, the LAS component competed least favorably for the surface over most of the composition range. Here it is the LS component that dominates at the adsorption, and as LS is the most hydrophobic its adsorption to the $\mathrm{A} / \mathrm{W}$ interface is a strongly energetically favorable process. However, the packing constraints imposed by the LS molecule also have a negative impact on the adsorption of LAS, and this is reflected in the patterns of adsorption seen in both the binary and ternary mixtures. The variations in the adsorption of the binary sophorolipid (LS and AS) and sophorolipid/LAS mixtures could be well described by ideal mixing or RST, but the trends observed in Figures 11 and 12 are outside the predictions of RST based on the behavior of the binary combinations. This further highlights the importance of the relative packing constraints on the surface mixing.

(c). Surface Structure of Sophorolipids and Sophorolipids Mixtures. The adsorption behaviors of mixtures of surfactants incorporating the sophorolipids appear to be significantly influenced by the packing constraints associated with the geometry of the sophorolipids and especially that of the LS component. Hence measuring the surface structure will provide some additional insights into the adsorption mechanism. Detailed structural information on the surface monolayer of the sophorolipids and sophorolipid mixtures have been obtained by the direct method of analysis based on the kinematic approximation, ${ }^{40}$ which provides information about the volume 
fraction distributions of the individually deuterium labeled components at the surface. In the kinematic approximation the reflectivity is given by

$$
R(Q)=\frac{16 \pi^{2}}{Q^{2}}\left[\sum_{i} b_{i}{ }^{2} h_{i i}+\sum_{i} \sum_{j<i} 2 b_{i} b_{j} h_{i j}\right]
$$

The $h_{i i}$ factors are the self-partial structure factors, $h_{i i}=$ $\left|\hat{n}_{i i}(Q)\right|^{2}$, the $h_{i j}$ cross-partial structure factors, are given by $h_{i j}(Q)=\operatorname{Re}\left\{\hat{n}_{i}(Q) \hat{n}_{j}(Q)\right\}$ and $\hat{n}_{i}(Q)$ is the one-dimensional Fourier transform of $\hat{n}_{i}(z)$. The self-partial structure factors relate directly to the distributions of the individual components at the interface, whereas the cross-partial structure factors relate to their relative positions at the interface, in the direction $(z)$ normal to the interface. This approach has been applied successfully to a range of different systems. ${ }^{29}$ Here the surfactant self-terms are represented as Gaussian distributions and the solvent distribution as a tanh function, ${ }^{41}$ as described in more detail in Annex 1 in the Supporting Information.

From the measurements for $1 \mathrm{mM} \mathrm{LS}$ and $1 \mathrm{mM}$ AS, for the isotopic combinations d-LS (AS)/NRW, d-LS (AS)/ $\mathrm{D}_{2} \mathrm{O}$, and h-LS (AS) $/ \mathrm{D}_{2} \mathrm{O}$, the three partial structure factors can be

Table 6. Model Parameters from $1 \mathrm{mM}$ LS and AS Partial Structure Analysis ${ }^{a}$

$\begin{array}{cccccc} & \sigma_{\mathrm{SL}} / \AA & n\left( \pm 0.02 \times 10^{-3}\right) / & \begin{array}{c}A / \AA^{2} \\ ( \pm 3)\end{array} & \begin{array}{c}\xi_{\mathrm{s}} / \AA \\ ( \pm 0.5)\end{array} & \begin{array}{c}\delta_{\mathrm{SLs}} / \AA \\ ( \pm 0.5)\end{array} \\ \text { system } & ( \pm 1) & \AA^{-2} & ( \pm 3) & & \\ \text { LS } & 12.0 & 1.35 & 70 & 2.0 & 7.0 \\ \text { AS } & 16.0 & 0.78 & 90 & 4.0 & 6.0\end{array}$

${ }^{a} \sigma_{i}$ and $n_{i}$ are the width and number density of the Gaussian describing the surfactant distribution, $\xi$ is the width of the tanh function describing the solvent distribution, and $\delta_{\text {is }}$ the separation between the solvent and surfactant distributions.

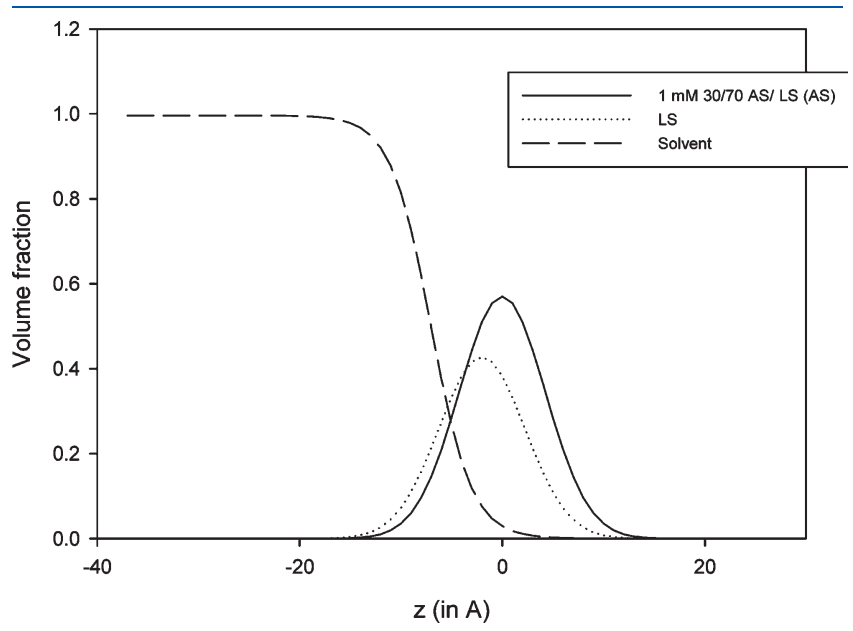

Figure 14. Volume fraction distributions for $1 \mathrm{mM} \mathrm{LS} / \mathrm{AS} 30 / 70 \mathrm{~mol}$ ratio at the $\mathrm{A} / \mathrm{W}$ interface, from partial structure factor analysis. extracted

$$
R(Q)=\frac{16 \pi^{2}}{Q^{2}}\left[b_{S L}{ }^{2} h_{S L_{S L}}+b_{s}{ }^{2} h_{s_{s}}+2 b_{S L} b_{s} h_{S L s}\right]
$$

The corresponding partial structure factors and the model fits for LS and AS are shown in Figure 8 in the Supporting Information. The resulting volume fraction distributions at the interface for the LS, the AS, and the solvent are plotted in figure 13.

The key model parameters from the analysis of the partial structure factors for LS and AS are summarized in Table 6.

The volume fraction distributions derived from the analysis of the partial structure factors and plotted in Figure 13 (the zero point in $z$ is arbitrarily chosen as the center of the surfactant distribution) show a number of important features. First the width of the LS (12 $\AA$ ) distribution is smaller than that for AS (16 $\AA$ ). This reflects the different conformation of the two molecules at the interface. The LS and the AS have the same sugar headgroups and hence this difference is associated with the alkyl chain structure, the AS having more extended chains than LS, as illustrated for the two different sophorolipid components in Figure 1. The solvent distribution is very narrow for LS ( $2 \AA$ ), implying that the headgroups of LS are more compact than the headgroups of AS. The solvent width in the presence of surfactant is usually broader than for the pure solvent and usually coincides with the headgroup distribution. This overlap is greater for AS ( $4 \AA$ ) than for LS ( $2 \AA)$. Finally, the position of the surfactant distribution relative to the solvent is broadly similar for LS and AS suggesting LS and AS occupy a similar position at the interface, but that the LS is less hydrated than the AS component.

For binary surfactant mixtures, six different isotopic combinations d-LS/h-AS/NRW, h-LS/d-AS/NRW, d-LS/d-AS/NRW, $\mathrm{d}-\mathrm{LS} / \mathrm{h}-\mathrm{AS} / \mathrm{D}_{2} \mathrm{O}$, h-LS $/ \mathrm{d}-\mathrm{AS} / \mathrm{D}_{2} \mathrm{O}$, and h-LS $/ \mathrm{h}-\mathrm{AS} / \mathrm{D}_{2} \mathrm{O}$ were measured at a surfactant concentration of $1 \mathrm{mM}$ and a solution composition of 30/70 mol ratio. The six partial structure factors

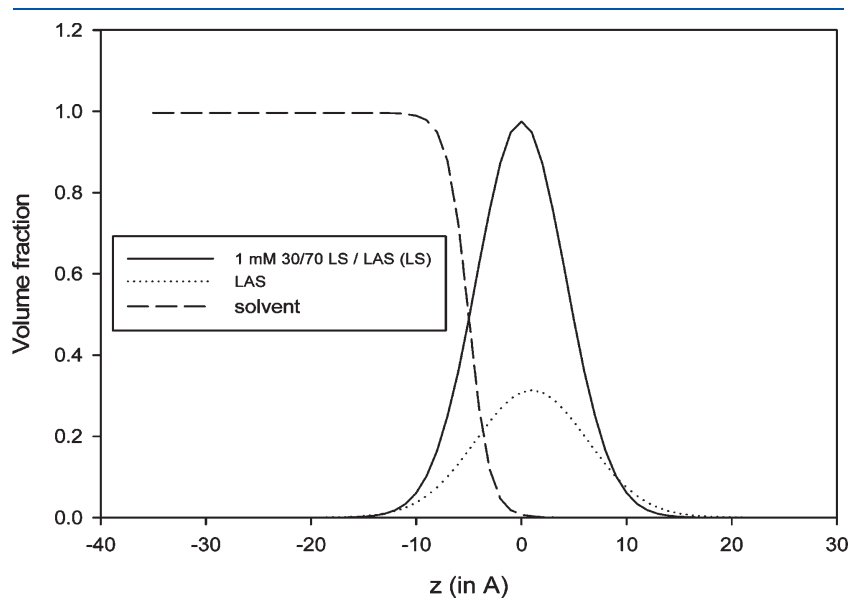

Figure 15. Volume fraction distributions for $1 \mathrm{mM} \mathrm{LS} / \mathrm{LAS} 30 / 70 \mathrm{~mol}$ ratio at the $\mathrm{A} / \mathrm{W}$ interface, from partial structure factor analysis.

\begin{tabular}{|c|c|c|c|c|c|c|c|c|c|}
\hline$\sigma_{\mathrm{LS}} / \AA ̊( \pm 1)$ & $\begin{array}{c}n_{\mathrm{LS}} 10^{-3} \AA^{-2} \\
( \pm 0.03)\end{array}$ & $\begin{array}{c}A_{\mathrm{LS}} / \AA^{2} \\
( \pm 3)\end{array}$ & $\begin{array}{c}\sigma_{\mathrm{AS}} / \AA \AA \\
( \pm 1)\end{array}$ & $\begin{array}{c}n_{\mathrm{AS}} 10^{-3} \AA^{-2} \\
( \pm 0.03)\end{array}$ & $\begin{array}{c}A_{\mathrm{AS}} / \AA^{2} \\
( \pm 10)\end{array}$ & $\begin{array}{c}\xi_{\mathrm{s}} / \AA \\
( \pm 0.5)\end{array}$ & $\begin{array}{l}\delta_{\mathrm{LSs}} / \AA \AA \\
( \pm 0.5)\end{array}$ & $\begin{array}{l}\delta_{\mathrm{ASs}} / \AA \AA \\
( \pm 0.5)\end{array}$ & $\begin{array}{c}\delta_{\text {LSAS }} / \AA \\
( \pm 0.5)\end{array}$ \\
\hline 12.0 & 0.45 & 210 & 12.0 & 0.6 & 155 & 4.0 & 7.0 & 5.0 & \\
\hline
\end{tabular}

Table 7. Model Parameters from 1 mM 30/70 LS/AS Partial Structure Factor Analysis 
Table 8. Model Parameters from 1 mM 30/70 LS/LAS Partial Structure Analysis

\begin{tabular}{|c|c|c|c|c|c|c|c|c|c|}
\hline $\begin{array}{c}\sigma_{\mathrm{LS}} \\
( \pm 1 \AA)\end{array}$ & $\begin{array}{l}n_{\mathrm{LS}} 10^{-3} \AA^{-2} \\
\quad( \pm 0.03)\end{array}$ & $\begin{array}{c}A_{\mathrm{LS}} \\
\left( \pm 3 \AA^{2}\right)\end{array}$ & $\begin{array}{c}\sigma_{\text {LAS }} \\
( \pm 1 \AA)\end{array}$ & $\begin{array}{c}n_{\text {LAS }} 10^{-3} \AA^{-2} \\
( \pm 0.03)\end{array}$ & $\begin{array}{c}A_{\mathrm{LAS}} / \AA^{2} \\
( \pm 10)\end{array}$ & $\begin{array}{c}\xi_{\mathrm{s}} / \AA \\
( \pm 0.5)\end{array}$ & $\begin{array}{l}\delta_{\mathrm{LSs}} / \AA \\
( \pm 0.5)\end{array}$ & $\begin{array}{c}\delta_{\mathrm{LASs}} / \AA \\
( \pm 0.5)\end{array}$ & $\begin{array}{c}\delta_{\text {LSLAS }} / \AA \AA \\
( \pm 0.5)\end{array}$ \\
\hline 12.0 & 1.3 & 72 & 15.0 & 0.55 & 140 & 1.0 & 5.0 & 6.0 & \\
\hline
\end{tabular}

in eq 5 are then

$$
\begin{aligned}
R(Q)= & \frac{16 \pi^{2}}{Q^{2}}\left[b_{L S}{ }^{2} h_{L S L S}+b_{A S}{ }^{2} h_{A S A S}+b_{s}{ }^{2} h_{s S}+2 b_{L S} b_{A S} h_{L S A S}\right. \\
& \left.+2 b_{L S} b_{s} h_{L S s}+2 b_{A S} b_{s} h_{A S S}\right]
\end{aligned}
$$

The six partial structure factor values have been determined and the resulting volume fraction distribution at the interface for LS/AS is plotted in Figure 14. The model parameters are summarized in Table 7 and the partial structure factors and associated model fits are shown in Figure 10 in the Supporting Information.

For $1 \mathrm{mM} 30 / 70 \mathrm{LS} / \mathrm{AS}$ mixtures, the self-terms are relatively weak (area/molecule $\sim 150,220$ respectively), and hence the determination of the cross-terms between the components is unreliable and has not been attempted. The solvent distribution is narrow, $\sim 4 \AA$, compared with the rhamnolipids/LAS system, where the solvent distribution is $\sim 7 \AA$, which is more typical of that encountered in conventional surfactant systems. ${ }^{29,40}$ This value is also comparable to the solvent distribution of the pure AS $\sim 4 \AA$ and is higher than that of the pure LS $\sim 2 \AA$, and it further reflects the highly compact and hydrophobic nature of the mixed surfactant monolayer.

For LS/LAS binary mixture, a similar set of measurements for the six different isotopic combinations, d-LS/d-LAS/NRW, d-LS/ h-LAS/NRW, h-LS/d-LAS/NRW, h-LS/h-LAS/D $2 \mathrm{O}$, d-LS/h$\mathrm{LAS} / \mathrm{D}_{2} \mathrm{O}$, and h-LS $/ \mathrm{d}-\mathrm{AS} / \mathrm{D}_{2} \mathrm{O}$ were made at a surfactant concentration of $1 \mathrm{mM}$ and a solution composition of $30 / 70$ mol ratio. The volume fraction distribution is plotted in Figure 15, and the key model parameters are summarized in Table 8 . The associated partial structure factors and the model fits are shown in Figure 11 in the Supporting Information.

The self-term for LS in LS/LAS mixture is the same as that for the pure LS $\sim 12 \AA$. It is small compared with rhamnolipids ${ }^{17}$ and other surfactant systems, ${ }^{29}$ which have typical values of $\sim 17 \AA$. This furthermore reflects the highly compact nature of this mixed surfactant monolayer. The solvent distribution is again narrow, and in this case is $\sim 1 \AA$. This value is very low compared with $7 \AA$ for rhamnolipids and implies a minimal amount of hydration.

In the structural measurements of the mixtures reported here, for LS/AS and LS/LAS, the structure of the LS component dominates the structure of the mixtures. As such the solvent distribution is narrow and the greater hydrophobicity of the LS substantially reduces the hydration of the mixed layer. With the exception of the LAS distribution the surfactant distribution is relatively narrow, reflecting the more compact structure of the LS component. These structural observations help to rationalize the greater surface activity of the LS and its dominance in the mixed adsorption. Furthermore in the ternary LS/AS/LAS mixtures rich in the AS component the structure of the AS will be closer to that of the LAS (as seen for AS alone) and so the dominant packing constraints of the LS component is partially compensated, resulting in a greater sophorolipid component in the adsorption.

Sun et al. ${ }^{42}$ discussed the effect of the chain length and degree of unsaturation of the fatty acid on the surface activity of the trehalolipids, and they reported that the CMC and area/molecule decreases sharply with the increase in the chain length and degree of unsaturation. For AS, Zhou et al demonstrated that the hydrophobic moiety of the AS is an unsaturated fatty acid with 1 to 2 double bonds and has a cis configuration. The double bond structure imposes a higher rigidity on the chain distribution and the cis configuration causes the chain to bend and to restrict the conformational freedom of the fatty acid even further. The more double bonds the chain has in the cis configuration, the less flexibility there is, and the molecule is correspondingly less watersoluble. For LS, the lactone is obtained by reacting the carboxyl group of the fatty acid with the $4^{\prime}$ hydroxyl group of the terminal glucose in sophorose through an esterification reaction, which means the lactone structure is even more rigid, more hydrophobic and consequently is even less water-soluble. These factors are reflected in the unusual aspects of the surface structures that are reported here, the dominance of the LS adsorption in the LS / AS/LAS mixtures, and in the packing constraints that are associated with that surface mixing.

\section{SUMMARY}

We have reported the use of surface tension and the neutron reflectivity, NR, to study the adsorption at the air/water interface of the sophorolipids, LS, AS and their mixtures. The deuterated and hydrogeneous sophorolipids used in this study were cultivated from a Candida bombicola culture, and separated and purified into their separate components. Compared with a related glycolipid, the rhamnolipids, ${ }^{25}$ the sophorolipids are slightly less surface active. The limiting surface tension of LS (diacetylated-C18:1) in UHQ water is $36 \mathrm{mN} / \mathrm{m}$ and the CMC is $6 \times 10^{-5} \mathrm{M}$. The limiting surface tension of AS (diacetylated-C18:1) in UHQ water is $38.5 \mathrm{mN} / \mathrm{m}$ with a CMC value of $6.7 \times 10^{-4} \mathrm{M}$. Both LS and AS have very rigid structures and the structural measurements indicate there is hardly any hydration of the compact LS monolayer. This structural rigidity may be attributed to the cisconfiguration of the alkyl chain, which normally contains one double bond. Furthermore for LS, the alkyl chain is in the form of a lactone ring which further restricts the molecular freedom, thus further reducing water solubility. The NR measurements show that LS dominates the surface in the binary mixtures of LS/AS and LS/LAS over the entire composition range measured. Although the surface is dominated by the LS component, the surface mixing is close to ideal. However, in the AS/LAS mixture the surface composition is closer to the solution composition and is broadly consistent with nonideal mixing as described by RST. In the ternary LS/AS/LAS mixtures the surface adsorption is also dominated by the sophorolipids, and especially the LS component. For the mixture LAS/LS/AS (1:2) the surface composition exhibits the most extreme partitioning in favor of the sophorolipids, and this is attributed to a reduction in the packing constraints at the surface due to the AS component. Unlike the rhamnolipids, ${ }^{16,17}$ the more extreme packing constraints associated with the sophorolipids inhibit any synergistic enhancement in the total surfactant adsorption in any of their related binary and ternary mixtures. 


\section{ASSOCIATED CONTENT}

S Supporting Information. Additional data on adsorbed amounts, tabulated model parameters from analysis of NR data, data plots, and partial structure factor plots not included in the main text. This material is available free of charge via the Internet at http://pubs.acs.org.

\section{AUTHOR INFORMATION}

\section{Corresponding Author}

*E-mail: jeff.penfold@stfc.ac.uk.

\section{ACKNOWLEDGMENT}

We acknowledge the provision of neutron beam time at ISIS (SURF) and at the ILL (FIGARO) and the invaluable assistance of the Instrument Scientists at ISIS and the ILL. The support of Unilever and the dti Technology programme for the project is acknowledged.

\section{REFERENCES}

(1) Deziel, E. P. G.; Villemur, R.; Lepione, F.; Bisaillon, J. Appl. Environ. Microbiol. 1996, 62 (6), 1908-1912.

(2) Ramana, K. V.; Karanth, N. G. J. Chem. Technol. Biotechnol. 1989, 45 (4), 249-257.

(3) Ratledge, C. In Biodeterioration, International Biodeterioration Symposium; Houghton, D. R., Ed.; Elsevier: Cambridge, U.K., 1987; pp 219-236.

(4) Hommel, R. K. Biodegradation 1990, 1 (2-3), 107-119.

(5) Beal, R.; Betts, W. B. J. Appl. Microbiol. 2000, 89 (1), 158-168.

(6) Soberon-Chavez, G.; Lepine, F.; Deziel, E. Appl. Microbiol. Biotechnol. 2005, 68 (6), 718-725.

(7) Lang, S.; Wagner, F. Biosurfactants: production, properties, applications; CRC Press: Boca Raton, FL, 1993.

(8) Zajic, J. E.; G., H.; Gerson, D. F. Biotechnol. Bioeng. 1977, 19 (9), $1303-1320$

(9) Georgiou, G. L.; Sung, C.; Sharma, M. M. Bio/Technology 1992, $10(1), 60-65$.

(10) Kretschmer, A.; Bock, H.; Wagner, F. Appl. Environ. Microbiol. 1982, 44 (4), 864-870.

(11) Koenig, S.; Quitzsch, K.; Koenig, B.; Hommel, R.; Haferburg, D.; Kleber, H. P. Colloids Surf., B: Biointerfaces 1993, 1 (1), 33-41.

(12) Zhu, Y.; Gan, J. J.; Zhang, G. L.; Yao, B.; Zhu, W. J.; Meng, Q. J. Zhejiang Univ., Sci., A 2007, 8 (9), 1514-1520.

(13) Rosenberg, E.; Ron, E. Z. Appl. Microbiol. Biotechnol. 1999, 52 (2), 154-162

(14) Sen, R. Prog. Energy Combust. Sci. 2008, 34 (6), 714-724.

(15) Banat, I. M.; Makkar, R. S.; Cameotra, S. S. Appl. Microbiol. Biotechnol. 2000, 53 (5), 495-508.

(16) Chen, M.; Penfold, J.; Thomas, R. K.; Smyth, T. J. P.; Perfumo, A.; Marchant, R.; Banat, I.; Stevenson, P.; Parry, A.; Tucker, I.; Grillo, I. Langmuir 2010, 26, 18281.

(17) Chen, M.; Penfold, J.; Thomas, R. K.; Smyth, T. J. P.; Perfumo, A.; Marchant, R.; Banat, I.; Stevenson, P.; Parry, A.; Tucker, I.; Grillo, I. Langmuir 2010, 26, 17958.

(18) Van Bogaert, I. N. A.; Saerens, K.; De Muynck, C.; Develter, D.; Soetaert, W.; Vandamme, E. J. Appl. Microbiol. Biotechnol. 2007, 76 (1), 23-34.

(19) Clenns, O. N.; Cooper, D. G. J. Am. Oil Chem. Soc. 2006, 83 (2), 137-145.

(20) Daverey, A.; Pakshirajan, K. Appl. Biochem. Biotechnol. 2009, $158(3), 663-674$

(21) Solaiman, D. K. Y.; Ashby, R. D.; Nunez, A.; Foglia, T. A. Biotechnol. Lett. 2004, 26 (15), 1241-1245.
(22) Otto, R. T.; Daniel, H. J.; Pekin, G.; Muller-Decker, K.; Furstenberger, G.; Reuss, M.; Syldatk, C. Appl. Microbiol. Biotechnol. 1999, 52 (4), 495-501.

(23) Zhang, L.; Somasundaran, P.; Singh, S. K.; Felse, A. P.; Gross, R. Colloids Surf. A-Physicochem. Eng. Aspects 2004, 240 (1-3), 75-82.

(24) Banat, I. M.; Smyth, T. J. P.; Perfumo, A.; Merchant, R.; Chen, M.; Penfold, J.; Thomas, R. K. Appl. Microbiol. Biotechnol. 2010, 87, 1347-1354

(25) Penfold, J.; Thomas, R. K.; Dong, C. C.; Tucker, I.; Metcalfe, K.; Golding, S.; Grillo, I. Langmuir 2007, 23 (20), 10140-10149.

(26) Penfold, J.; Richardson, R. M.; Zarbakhsh, A.; Webster, J. R. P.; Bucknall, D. G.; Rennie, A. R.; Jones, R. A. L.; Cosgrove, T.; Thomas, R. K.; Higgins, J. S.; Fletcher, P. D. I.; Dickinson, E.; Roser, S. J.; McLure, I. A.; Hillman, A. R.; Richards, R. W.; Staples, E. J.; Burgess, A. N.; Simister, E. A.; White, J. W. J. Chem. Soc.-Faraday Trans. 1997, 93 (22), 3899-3917.

(27) FIGARO reflectometer at ILL. http://www.ill.eu/instrumentssupport/instruments-groups/instruments/figaro/ (18 January 2010).

(28) Lee, E. M.; Thomas, R. K.; Penfold, J.; Ward, R. C. J. Phys. Chem. 1989, 93 (1), 381-388.

(29) Lu, J. R.; Thomas, R. K.; Penfold, J. Adv. Colloid Interface Sci. 2000, 84 (1-3), 143-304.

(30) Staples, E.; Thompson, L.; Tucker, I.; Penfold, J.; Thomas, R. K.; Lu, J. R. Langmuir 1993, 9 (7), 1651-1656.

(31) Penfold, J.; Staples, E.; Thompson, L.; Tucker, I.; Hines, J.; Thomas, R. K.; Lu, J. R. Langmuir 1995, 11 (7), 2496-2503.

(32) Tucker, I.; Penfold, J.; Thomas, R. K.; Tildesley, D. J. Langmuir 2009, 25 (7), 3924-3931.

(33) Matsuda, T.; Asoh, Y.; Villeneuve, M.; Matsubara, H.; Takiue, T.; Aratono, M. Colloid Polym. Sci. 2004, 282 (4), 324-329.

(34) Hines, J. D.; Thomas, R. K.; Garrett, P. R.; Rennie, G. K.; Penfold, J. J. Phys. Chem. B 1997, 101 (45), 9215-9223.

(35) Born, M.; Wolf, E.; Bhatia, A. B. Principles of optics: electromagnetic theory of propagation, interference and diffraction of light, 7 th ed.; Cambridge University Press: Cambridge, U.K., 1999.

(36) Holland, P. M. Colloids Surf. 1986, 19, 171.

(37) Penfold, J.; Staples, E.; Thompson, L.; Tucker, I. Colloids Surf. A-Physicochem. Eng. Aspects 1995, 102, 127-132.

(38) Penfold, J.; Staples, E.; Tucker, I.; Thomas, R. K.; Woodling, R.; Dong, C. C. J. Colloid Interface Sci. 2003, 262 (1), 235-242.

(39) Hines, J. D.; Garrett, P. R.; Rennie, G. K.; Thomas, R. K.; Penfold, J. J. Phys. Chem. B 1997, 101 (36), 7121-7126.

(40) Lu, J. R.; Li, Z. X.; Smallwood, J.; Thomas, R. K.; Penfold, J. J. Phys. Chem. 1995, 99 (20), 8233-8243.

(41) Lu, J. R.; Hromadova, M.; Simister, E. A.; Thomas, R. K.; Penfold, J. J. Phys. Chem. 1994, 98 (44), 11519-11526.

(42) Sun, Y.; Xia, W.; Tang, X.; He, Z.; Chen, J. Frontiers Chem. Eng. China 2009, 3 (4), 407-412.

(43) Zhou, S. Q.; Xu, C.; Wang, J.; Gao, W.; Akhverdiyeva, R.; Shah, V.; Gross, R. Langmuir 2004, 20 (19), 7926-7932. 